\title{
Intrinsic Cornu Ammonis Area 1 Theta-Nested Gamma Oscillations Induced by Optogenetic Theta Frequency Stimulation
}

\author{
James L. Butler, Philipe R. F. Mendonça, Hugh P. C. Robinson, and $₫$ Ole Paulsen \\ Department of Physiology, Development and Neuroscience, University of Cambridge, Physiological Laboratory, Cambridge CB2 3EG, United Kingdom
}

Gamma oscillations $(30-120 \mathrm{~Hz})$ are thought to be important for various cognitive functions, including perception and working memory, and disruption of these oscillations has been implicated in brain disorders, such as schizophrenia and Alzheimer's disease. The cornu ammonis area 1 (CA1) of the hippocampus receives gamma frequency inputs from upstream regions (cornu ammonis area 3 and medial entorhinal cortex) and generates itself a faster gamma oscillation. The exact nature and origin of the intrinsic CA1 gamma oscillation is still under debate. Here, we expressed channelrhodopsin-2 under the CaMKII $\alpha$ promoter in mice and prepared hippocampal slices to produce a model of intrinsic CA1 gamma oscillations. Sinusoidal optical stimulation of CA1 at theta frequency was found to induce robust theta-nested gamma oscillations with a temporal and spatial profile similar to CA1 gamma in vivo. The results suggest the presence of a single gamma rhythm generator with a frequency range of $65-75 \mathrm{~Hz}$ at $32^{\circ} \mathrm{C}$. Pharmacological analysis found that the oscillations depended on both AMPA and $\mathrm{GABA}_{\mathrm{A}}$ receptors. Cell-attached and whole-cell recordings revealed that excitatory neuron firing slightly preceded interneuron firing within each gamma cycle, suggesting that this intrinsic CA1 gamma oscillation is generated with a pyramidal-interneuron circuit mechanism.

Key words: circuit; hippocampus; interneuron; mouse; network; oscillation

\section{Significance Statement}

This study demonstrates that the cornu ammonis area 1 (CA1) is capable of generating intrinsic gamma oscillations in response to theta input. This gamma generator is independent of activity in the upstream regions, highlighting that CA1 can produce its own gamma oscillation in addition to inheriting activity from the upstream regions. This supports the theory that gamma oscillations predominantly function to achieve local synchrony, and that a local gamma generated in each area conducts the signal to the downstream region.

\section{Introduction}

The timing of neural cell firing is thought to be essential for successful information coding within the brain. Gamma oscillations, rhythmic activity measured in the local field potential (LFP) with a frequency range of $30-120 \mathrm{~Hz}$, synchronize the

Received Aug. 21, 2015; revised Feb. 14, 2016; accepted Feb. 18, 2016.

Author contributions: J.L.B., P.R.F.M., H.P.C.R., and O.P. designed research; J.L.B. and P.R.F.M. performed research; J.L.B. analyzed data; J.L.B., P.R.F.M., H.P.C.R., and 0.P. wrote the paper.

This work was supported by a Biotechnology and Biological Sciences Research Council CASE Studentship in collaboration with Eli Lilly and Company (to J.L.B.), and by Coordenação de Aperfeiçoamento de Pessoal de Nível Superior Science without Borders Cambridge Scholarship (to P.R.F.M.).

The authors declare no competing financial interests.

This article is freely available online through the J Neurosci Author Open Choice option.

Correspondence should be addressed to Ole Paulsen at the above address. E-mail: op210@cam.ac.uk.

DOI:10.1523/JNEUROSCI.3150-15.2016

Copyright $(2016$ Butler et al.

This is an Open Access article distributed under the terms of the Creative Commons Attribution License Creative Commons Attribution 4.0 International, which permits unrestricted use, distribution and reproduction in any medium provided that the original work is properly attributed. spiking activity of neural populations to a specific phase of the gamma wave, thus segregating cell firing into the different gamma cycles (Buzsáki and Wang, 2012). Gamma oscillations often co-occur with theta oscillations $(4-12 \mathrm{~Hz})$ to which they are phase-amplitude coupled, such that the gamma oscillations have a larger amplitude at a consistent specific phase of the theta cycle (Buzsáki et al., 1983). This complex temporal control of neural activity is thought to be important for cognitive tasks, including navigation, sensory association, and working memory. Disruption of gamma oscillations has been implicated in a diverse range of diseases, such as schizophrenia (Uhlhaas and Singer, 2010) and Alzheimer's disease (Goutagny and Krantic, 2013). It is therefore important to understand the mechanisms underlying gamma oscillations.

The cornu ammonis area 1 (CA1) of the hippocampus exhibits multiple types of gamma oscillations in vivo, which can be segregated based on their spectral, temporal, and spatial profiles. A gamma oscillation in the range of $30-80 \mathrm{~Hz}$ originates in cornu ammonis 
area 3 (CA3) and propagates along the Schaffer collaterals to the stratum radiatum (SR) of the CA1 (Csicsvari et al., 2003). In the medial entorhinal cortex ( $\mathrm{mEC}$ ), a faster gamma oscillation is generated in the range of $60-120 \mathrm{~Hz}$ and propagates to the terminal region of $\mathrm{mEC}$ projections in the CA1, the stratum lacunosummoleculare (SLM; Colgin et al., 2009). Both of these afferent gamma oscillations can also be recorded from the stratum pyramidale (SP), the location of the cell bodies of CA1 pyramidal neurons, as well as from their respective input layers. Using independent component analysis, a third gamma oscillation generated in the SP was recently identified in the CA1 (Schomburg et al., 2014). This locally generated gamma oscillation has a similar frequency $(>100 \mathrm{~Hz})$ to $\mathrm{mEC}$ gamma, but it has a distinct temporal and spatial profile, suggesting the presence of a gamma generator in the CA1 that is independent of the afferent gamma generators.

In vitro preparations have been put to good use to reveal the mechanisms underlying the current generators for hippocampal oscillations (for review, see Butler and Paulsen, 2015). CA3 gamma oscillations can be induced in acute hippocampal slices by application of the cholinergic agonist carbachol (Fisahn et al., 1998) and $\mathrm{mEC}$ gamma oscillations have been induced by optogenetic excitation of stellate cells in the mEC (Pastoll et al., 2013). In the CA1, strong excitation by tetanic electrical stimulation (Whittington et al., 1995) or high concentrations of kainate (Traub et al., 2003) can give rise to local CA1 gamma. More recently, it was found that when the connections between CA1 and CA3 are severed, carbachol induces CA1 gamma oscillations (Pietersen et al., 2014). However, CA1 gamma oscillations in more physiological conditions, such as in the presence of theta oscillations, have not yet been reported in the intact hippocampal slice.

Here, we asked whether sinusoidally modulated theta-frequency optogenetic stimulation of the CA1 is sufficient to generate gamma oscillations in acute hippocampal slices. Expressing the optogenetic activator channelrhodopsin-2 (ChR2) under the CaMKII $\alpha$ promoter in excitatory neurons granted us high temporal and spatial control over the pyramidal cells of the CA1. We found that sinusoidally modulated stimulation in the theta frequency range induced robust gamma oscillations, which were phase-amplitude coupled to the theta stimulation. Both the frequency and theta coupling were analogous to those seen for CA1 gamma oscillations in vivo. The oscillations were resilient to changes in the input to the CA1 region and pharmacological analysis found that the oscillations depended on both AMPA and $\mathrm{GABA}_{\mathrm{A}}$ receptors. Cell-attached and wholecell recordings revealed that excitatory neuron firing slightly preceded interneuron firing within each gamma cycle, confirming a pyramidal-interneuron circuit mechanism of gamma generation.

\section{Materials and Methods}

Mice. Transgenic mice expressing the optogenetic activator ChR2 under the CaMKII $\alpha$ promoter were created by crossing LoxP-ChR2(H134R)EYFP $^{+/+}$mice on a 129 S6 background (Jackson Laboratories, stock $\# 012569$; http://jaxmice.jax.org/strain/012569.html) with CaMKII $\alpha$ $\mathrm{Cre}^{+/+}$mice on a C57BL/6 background (Jackson Laboratories, stock \#005359; http://jaxmice.jax.org/strain/005359.html). This resulted in heterozygous mice expressing both CaMKII $\alpha$-Cre and LoxPChR2(H134R)-EYFP, thus expressing ChR2 selectively in CaMKII $\alpha$ cells, including pyramidal cells in the CA1 of the hippocampus. All animal care and experimental procedures were conducted in accordance with the United Kingdom Animals (Scientific Procedures) Act of 1986. All mice used were between 4 and 8 weeks old, and both genders were used.

In vivo recordings. Adult mice of the C57BL/6 strain $(n=4)$ were anesthetized with $2 \mathrm{~g} / \mathrm{kg}$ urethane (Sigma-Aldrich; diluted 20\% w/v in saline) and supplemented with $0.2 \mathrm{~g} / \mathrm{kg}$ urethane as needed (approximately twice per $4 \mathrm{~h}$ session). Mice were then placed in a stereotaxic frame and the skin and skull above the hippocampus removed. An extracellular recording electrode was then slowly inserted into the CA1 SP of the right hippocampus. Tail pinches were used to induce activity and recordings taken immediately after tail pinching were used for analysis. The recording site was confirmed post hoc by passing a $3 \mathrm{~mA}$ current for $2 \mathrm{~s}$ through the recording electrode, making acute slices from the brain, and then identifying the lesion site under a microscope.

Slice preparation. The transgenic mice were deeply anesthetized with $4 \%(\mathrm{v} / \mathrm{v})$ isoflurane at $1.4 \mathrm{~L} \cdot \mathrm{min}^{-1}$ and decapitated. The brain was then quickly removed and horizontal (unless otherwise stated) hippocampal slices with a thickness of $350-400 \mu \mathrm{m}$ were prepared in cold $\left(0-3^{\circ} \mathrm{C}\right)$, oxygenated $\left(95 \% \mathrm{O}_{2}, 5 \% \mathrm{CO}_{2}\right)$ artificial CSF (aCSF) containing the following: $126 \mathrm{~mm} \mathrm{NaCl}, 3 \mathrm{~mm} \mathrm{KCl}, 1.25 \mathrm{~mm} \mathrm{NaH}_{2} \mathrm{PO}_{4}, 2 \mathrm{~mm} \mathrm{MgSO}_{4}, 2 \mathrm{~mm}$ $\mathrm{CaCl}_{2}, 26.4 \mathrm{~mm} \mathrm{NaHCO}_{3}$, and $10 \mathrm{~mm}$ glucose, $\mathrm{pH}$ 7.2. Slices were stored in a submerged-style storage chamber at room temperature $\left(22-24^{\circ} \mathrm{C}\right)$ for $\geq 50 \mathrm{~min}$ before use. For minislice preparation, slices were gently removed from the recording chamber and a cut was made under a microscope. The slice was then remounted in the recording chamber and left to recover for $10 \mathrm{~min}$.

Multielectrode recordings. Multielectrode probes were coated in polyD-lysine for $\geq 1$ min to aid with slice adhesion and then rinsed with aCSF. Probes contained 64 electrodes $50 \mu \mathrm{m}$ in size, structured in an even $8 \times$ 8 grid with an interpolar distance of $150 \mu \mathrm{m}$ (Panasonic MED-P5155, Tensor Biosciences). Slices were mounted to incorporate as much of the $\mathrm{CA} 1$ as possible. Slices were then transferred to the recording unit, where they were maintained in a submerged condition, continually superfused with aCSF at a rate of $3-4 \mathrm{ml} \cdot \mathrm{min}^{-1}$ and heated to $27-29^{\circ} \mathrm{C}$. The slices were left for $\geq 10 \mathrm{~min}$ before recording commenced at a $20 \mathrm{kHz}$ acquisition rate using the Panasonic MED64 system (Tensor Biosciences).

Patch-clamp recordings. Single-cell recordings were performed in slices, using a Multiclamp 700B amplifier and CV-7B headstages (Molecular Devices). Signals were acquired at $20 \mathrm{kHz}$ (4 kHz Bessel filter) with custom Matlab software controlling a 16 bit National Instruments analog board. Slices were superfused with aCSF at $27-29^{\circ} \mathrm{C}$. Before patching, a borosilicate glass electrode (3-5 M $\Omega$ ) filled with 10 mM HEPES-buffered aCSF for LFP recording was positioned in the SP close to the cell that was patched. All pyramidal neurons recorded from were in the SP and all interneurons from which recordings were made were in either the SP or the stratum oriens ( $\mathrm{SO}$ ).

Three different patch-clamp configurations were used to verify the input and output of individual cells during theta-nested gamma oscillations. To examine the output of cells, spikes from single neurons were recorded in cell-attached voltage-clamp mode followed by whole-cell current-clamp mode. First, a borosilicate glass pipette (3-5 M $\Omega$ ) filled with HEPES-buffered aCSF was used to create a low-resistance seal (30$500 \mathrm{M} \Omega$, which would later sometimes develop to a gigaseal). After the completion of the recording, the same cell was repatched in whole-cell current-clamp mode with a 5-7 M $\Omega$ pipette containing the following: $105 \mathrm{~mm}$ K gluconate, $30 \mathrm{~mm} \mathrm{KCl}, 10 \mathrm{~mm}$ HEPES, $10 \mathrm{~mm}$ phosphocreatine $\mathrm{Na}_{2}, 4 \mathrm{~mm}$ ATP $\mathrm{Mg}$, and $0.3 \mathrm{~mm}$ GTP $\mathrm{Na}(-10 \mathrm{mV}$ liquid junction potential correction).

To examine the input to cells, IPSCs and EPSCs were recorded in whole-cell voltage-clamp mode with the following intracellular solution: $130 \mathrm{~mm}$ Cs methanesulfonate, $3 \mathrm{~mm} \mathrm{CsCl}, 10 \mathrm{~mm}$ HEPES, 1 mM EGTA, 10 mM phosphocreatine $\mathrm{Na}_{2}, 4 \mathrm{~mm}$ ATP $\mathrm{Mg}, 0.3 \mathrm{~mm}$ GTP Na, and $5 \mathrm{~mm}$ QX314, pH 7.2 (balanced with $\mathrm{CsOH},-8 \mathrm{mV}$ liquid junction potential correction). During optical stimulation, IPSCs were recorded at $-10 \mathrm{mV}$ and EPSCs at $-70 \mathrm{mV}$. Series resistance was always $<35 \mathrm{M} \Omega$ and compensated up to $90 \%$.

Neurobiotin $(0.5 \%)$ was also added to the intracellular solution. Neurobiotin allowed cells to be stained by Alexa Fluor 633 streptavidin (Life Technologies) and have their morphologies confirmed. The cells were left in whole-cell mode for $\geq 15$ min to allow a sufficient amount of neurobiotin to enter the cell.

Staining. After a successful cell recording had taken place, the slice was transferred to a $4 \%$ paraformaldehyde solution and left overnight. The next day, the slice was thoroughly washed in PBS solution and then left in Alexa 
Fluor 633 streptavidin (Life Technologies) for $3 \mathrm{~h}$. The slice was then washed once again using PBS solution and fixed on a microscope slide. The slides were then left in darkness at $4^{\circ} \mathrm{C}$ for $\geq 24 \mathrm{~h}$ before being imaged.

Imaging. A Leica SP2 confocal microscope was used to image the cells that had been recorded. The following fluorescence excitation and emission wavelengths were used to visualize the slices: DAPI, 405 and $420-$ $460 \mathrm{~nm}$; enhanced yellow fluorescent protein (eYFP), 514 and 530-560 $\mathrm{nm}$; Alexa Fluor 633, 633 and $640-700 \mathrm{~nm}$. Cells were first visualized at $10 \times$ resolution and this was then increased to $63 \times$ if a putative interneuron was identified for further confirmation of the cell type. For each cell, a minimum $2-\mu \mathrm{m}$-resolution $z$-stack was acquired, which was used for 3D reconstructions with the software Vaa3D (Peng et al., 2010, 2014). Cells were classified as pyramidal neurons when the stereotypical apical dendrite was observed, and interneurons when only local projections were identified.

Light delivery. A $473 \mathrm{~nm}$ diode-pumped solid-state laser (Ciel, Laser Quantum) or a $470 \mathrm{~nm}$ digital micromirror device (Polygon, Mightex) was used to excite ChR2. Light was delivered to the top of the slice through the objective of an Olympus BX51 microscope. The light, which covered a $500-\mu \mathrm{m}$-diameter circle or a $500 \times 500 \mu \mathrm{m}$ square in the case of the laser or digital micromirror device respectively, was positioned over the center of the CA1. This resulted in illumination covering the majority of the SP and the SR and approximately half of the SO and the SLM. Igor Pro (Wavemetrics) or Matlab (Mathworks) was used to control the light sources, modulating the light sinusoidally at a frequency of $5 \mathrm{~Hz}$ and synchronizing the light stimulation with the recordings. In all cases, the maximum light intensity $(100 \%)$ was $<2.5 \mathrm{~mW} \cdot \mathrm{mm}^{-2}$.

Pharmacology. Stock solutions $(1000 \times)$ of the drugs were made using the following solvents: dimethyl sulfoxide (DMSO) for the GluK1subunit-containing kainate receptor antagonist UBP-302, the $\mathrm{GABA}_{\mathrm{A}}$ receptor antagonist $(+)$-bicuculline, the AMPA and kainate receptor antagonist 2,3-dihydroxy-6-nitro-7-sulfamoyl-benzo[f] quinoxaline-2, 3-dione (NBQX) and the allosteric AMPA receptor modulator cyclothiazide (CTZ); ethanol for the barbiturate ( \pm )-thiopental; 1.1 molar equivalent $\mathrm{NaOH}$ for the metabotropic glutamate (mGlu) receptor antagonist (RS)- $\alpha$-methyl-4-carboxyphenylglycine (MCPG); $\mathrm{H}_{2} \mathrm{O}$ for the muscarinic receptor antagonist atropine, the $N$-methyl-D-aspartate (NMDA) receptor antagonist DL-2-amino-5-phosphonopentanoic acid (AP5), and the AMPA receptor antagonist GYKI-53655. These stock solutions were then frozen, defrosted on the morning of the relevant experiment, and diluted $1000 \times$ in aCSF. After a 20 min incubation period, a recording of activity was taken during stimulation with blue light. The relevant drug solution was then bath applied and another recording taken 10 min later. All drugs and reagents were purchased from either Tocris Bioscience or Sigma-Aldrich.

Data analysis and statistics. Data were imported into Igor Pro (Wavemetrics), where all data analysis was conducted using custom scripts. For each recording, Welch's power spectral density (PSD) was calculated. The peak value in the range of $30-100 \mathrm{~Hz}$ was used to calculate the peak gamma frequency and the area under the PSD $\pm 15 \mathrm{~Hz}$ from the peak was used as a measure of the power of the gamma oscillations. No physiological signals were observed above this frequency range. Some recordings had a peak gamma frequency of $30 \mathrm{~Hz}$ due to contamination by theta activity, and were therefore excluded from further analysis. For the multielectrode recordings, the aforementioned process was done for each of the 64 electrodes and the channel with the highest power in the SP (unless otherwise stated) was chosen for further analysis.

For phase calculations, a continuous wavelet transform (CWT) for each theta cycle was calculated using a normalized Morlet wavelet transform $\left(\omega_{0}=8\right)$. This allowed for accurate automated detection and extraction of gamma activity without the need for bandpass filtering. For the pharmacology experiments, when no drug was added to the aCSF, there was a rundown of the power of the gamma oscillations during the $10 \mathrm{~min}$ incubation period. The change in power and frequency for the different drugs was therefore compared, using an independent samples two-tailed Student's $t$ test, with the condition when no drug was added. The cell-spiking experiments were also compared using an independent samples two-tailed Student's $t$ test. A paired Student's $t$ test was used for the minislice and layer-specific stimulation experiments. As multiple comparisons were also made, $p$ value thresholds were Bonferroni corrected to compensate for this (slice angles and minislices, $0.05 / 2=0.025$; layer-specific stimulation, $0.05 / 3=0.0167$; pharmacology, $0.05 / 10=$ $0.005)$. All averages presented are mean \pm SEM or the mean angle and $r$ value in the case of circular data.

For the current source density (CSD) profile, cycle averages for each channel were constructed from 48 theta cycles. These averages were bandpass filtered between 30 and $120 \mathrm{~Hz}$ to isolate the gamma component of the oscillation. They were then smoothed using a $3 \times 3$ Gaussian filter and convolved with a $3 \times 3$ Laplacian filter $(0-10,-14-1,0-1$ $0)$ to attain the second spatial derivative. Signals from the outer 28 electrodes are not presented to avoid edge artifacts.

To correlate intracellular recordings to extracellular LFP, a threshold paradigm was used to isolate single-cell events. Each theta cycle was meansubtracted, accounting for any drift that occurred over the length of a recording session. For cell-attached recordings, spikes of amplitude $>3$ SDs above the mean were included. For voltage-clamp recordings, positive or negative deflections (for IPSCs or EPSCs, respectively) $>30 \mathrm{pA}$ were extracted. The maximum (for IPSCs) or minimum (for cell-attached spikes and EPSCs) was then used as the time reference point for phase calculation. A CWT was constructed for the concurrent LFP and used to identify the gamma peak and trough flanking the single-cell event. Only cases where the magnitude of the instantaneous gamma cycle was two SDs above the average of the entire frequency range tested $(1-100 \mathrm{~Hz})$ were included. The position of the spike or EPSC between the two adjacent troughs was then calculated to give the phase value for each data point. For testing whether phase data were significantly nonuniform, Rayleigh's test for uniformity was used. The mean angle and accompanying $r$ value (which represents the strength of phase preference, with 0 being no preference and 1 being a single unimodal direction) was then calculated to assess the direction and strength of any phasephase coupling.

\section{Results}

Optogenetic stimulation of the CA1 activates a single intrinsic gamma oscillation

When recording the LFP in the SP from the CA1 of a urethaneanesthetized mouse, a tail pinch consistently induced network activity that persisted for a few seconds afterward (Fig. 1A, top). From the PSD, this network activity consisted of two main components: a large amplitude theta oscillation and a smaller amplitude gamma component spanning the majority of the gamma range (Fig. 1B). When only the theta cycles containing fast gamma cycles $(>50 \mathrm{~Hz})$ were analyzed, the oscillations were phase-locked to the trough of the theta cycle measured in the LFP (Fig. 1C).

By expressing ChR2 in the CA1 and preparing acute hippocampal slices, we were able to induce CA1 gamma oscillations in vitro in response to stimulation with a $2.5 \mathrm{~mW} \cdot \mathrm{mm}^{-2}$ blue light sinusoidally modulated at a frequency of $5 \mathrm{~Hz}$ (Fig. $1 A$, bottom). In contrast to the in vivo condition, these oscillations had a narrow, specific frequency range $\left(52 \pm 1 \mathrm{~Hz}\right.$ at $27-28^{\circ} \mathrm{C}, n=106$ slices; Fig. $\left.1 B\right)$, which was consistent between all slices and animals tested. As was the case for in vivo gamma oscillations, the in vitro oscillations were phase-amplitude coupled to the theta stimulation, occurring at the peak of each sinusoidal stimulation cycle (the trough of the theta activity recorded in the LFP; Fig. 1C).

The oscillations were stable over time (Fig. 1D) and persisted for as long as the light stimulation continued, immediately stopping when the light stimulation did (Fig. 1E). The oscillations could be induced using slices prepared using all three different angles tested (horizontal, coronal, and longitudinal), with no difference in the power (Fig. $1 F$ ) or frequency (Fig. $1 G$ ) between the different slicing angles. When the temperature of the superfusion solution was increased, the in vitro gamma oscillations decreased in power by $78 \pm 3 \%$ from room temperature $\left(22-24^{\circ} \mathrm{C}\right)$ to $32^{\circ} \mathrm{C}$ (Fig. $1 H$ ). The frequency of the oscillations was found to have the opposite relationship with temperature, increasing from $35 \pm 1$ 
A
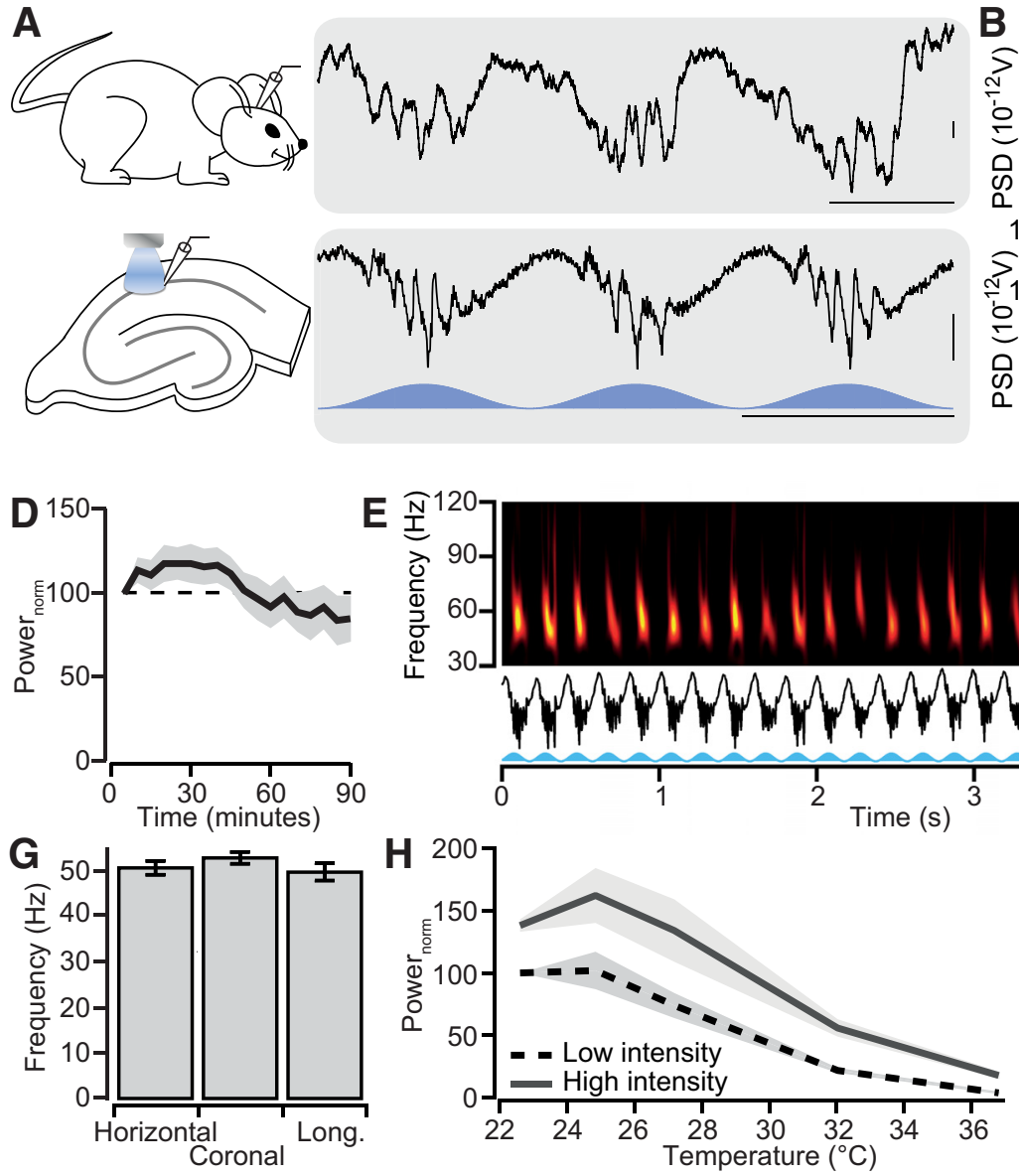

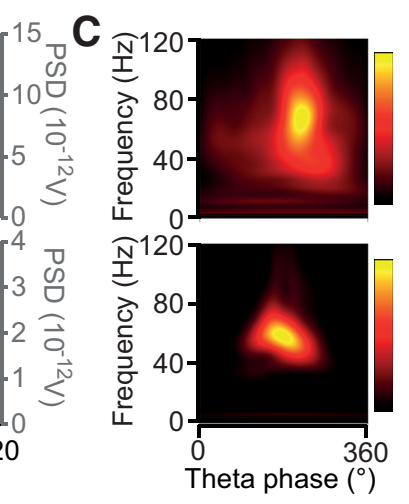

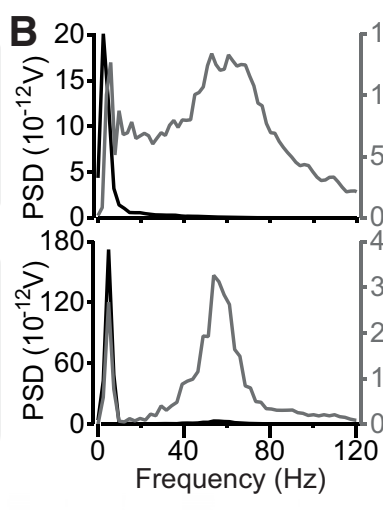
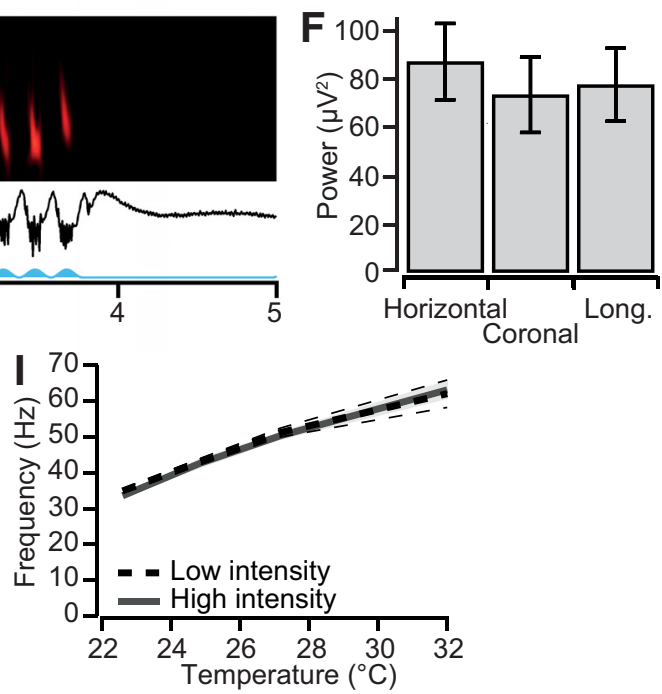

Figure 1. Optogenetic induction of CA1 gamma in hippocampal slices expressing ChR2. A, Representative traces of extracellular field activity recorded in the SP from both an anesthetized head-fixed wild-type mouse (in vivo, top) and from acute brain slices expressing ChR2 in the CA1 (in vitro, bottom; blue trace represents the optical stimulation). Vertical scale bar, $50 \mu$; ; horizontal scale bar, $200 \mathrm{~ms}$. B, PSD plots of the activity recorded from the data shown in $\boldsymbol{A}$ for both the in vivo (top) and in vitro (bottom) recordings. The black line is the power for the wide bandpass-filtered trace where the dominant theta peak is clearly visible, and gray line is the power with a $30-120 \mathrm{~Hz}$ bandpass filter applied to the recordings. $\mathbf{C}$, CWTs allow for comparison of the phase of the oscillations. Peaks in the theta range were used to divide the recordings into individual theta bins and the average scalogram of all theta bins extracted ( $n=50$ ) is displayed for in vivo (top) and in vitro (bottom) conditions. $\boldsymbol{D}$, The average changes in the power of the gamma oscillations (sampled for 10 s every 5 min) over a 90 min recording period. $\boldsymbol{E}$, The gamma oscillations were stable between theta cycles and stopped when the optical stimulation was stopped. $\boldsymbol{F}$, Gamma oscillations could be induced from all three slice angles tested. The average power of the gamma elicited is displayed for slices prepared with a horizontal, coronal, or longitudinal (Long.) angle. $n=22$ for horizontal and coronal angles and $n=11$ for longitudinal slice angles. $\boldsymbol{G}$, Same as in $\boldsymbol{F}$ but the average frequency is plotted. For both $\boldsymbol{F}$ and $\boldsymbol{G}$, there were no significant differences between either of the different slice angles compared with the horizontal angle when assessed using an independent samplest test with Bonferroni's corrections ( $p$ value threshold, 0.025$)$. $\boldsymbol{H}$, The effect of temperature on the power of the gamma oscillations at two different stimulation intensities $(n=$ 11). $\boldsymbol{I}$, Same as in $\boldsymbol{H}$ but for the frequency of the oscillations produced.

$\mathrm{Hz}$ at room temperature $\left(22-24^{\circ} \mathrm{C}\right)$ to $62 \pm 4 \mathrm{~Hz}$ at $32^{\circ} \mathrm{C}(n=11$; Fig. $1 I)$. Increasing the intensity of light stimulation failed to revert the changes in frequency, demonstrating this effect was not caused by a temperature-dependent reduction in ChR2 kinetics (Fig. 1I). There were no discernible oscillations present above $32^{\circ} \mathrm{C}$ and this was accompanied by a severe reduction in both excitatory and inhibitory currents in CA1 cells (data not shown). This was presumably due to the high metabolic strain that oscillations place on in vitro slices (Hájos et al., 2009).

To accurately localize the sinks and sources of the optogenetic gamma across the CA1, we conducted a CSD analysis. Recording from an $8 \times 8$ electrode grid allowed us to visualize activity across the majority of the CA1 during stimulation. The gamma oscillations were present in both the SP and SLM layers, with no activity observed in the intermediary SR layer. The oscillations spread $\sim 400 \mu \mathrm{m}$ into the nearby areas of the dentate gyrus and subiculum, where they could be detected in the LFP and decreased in magnitude with distance from the CA1 (data not shown). Much smaller theta-nested gamma oscillations could also be detected in the deep layers of the mEC during CA1 stimulation, though it is unclear whether these were due to volume conduction through the extracellular tissue or generated locally by CA1 projections that terminate in these layers of the mEC.

Cycle averages were constructed from 48 theta cycles and both the SP and SLM gamma cycles were still clearly visible after this averaging, demonstrating the strong phase preference between theta cycles of the oscillations (Fig. 2C). The CSD showed large uniform sink and source bands across the transverse axis of the SP and the SLM, demonstrating that the entirety of the CA1 from which recordings were taken participated synchronously in the oscillations. This again suggested that when the CA1 receives theta input, a single gamma generator is activated that entrains all connected cells of the CA1 to this specific oscillation (Fig. 2D). Furthermore, the 2D CSD revealed that the SP and the SLM were an alternating sink-source pair (Fig. 2D), suggesting that the CA1 is the location of the current generator for the oscillations and that, therefore, these oscillations do not rely on input from the upstream regions. 

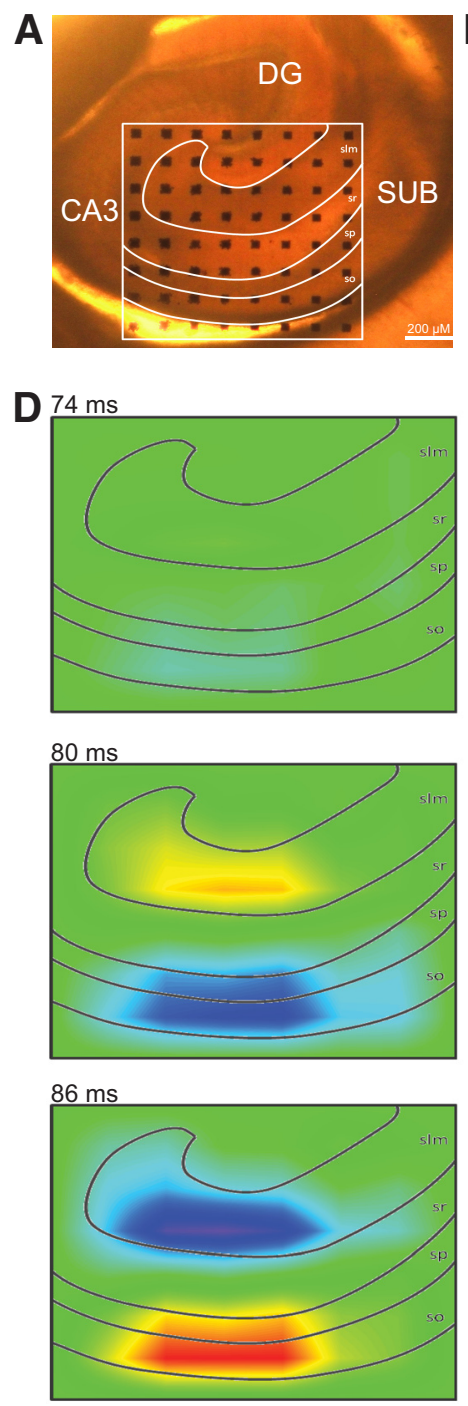
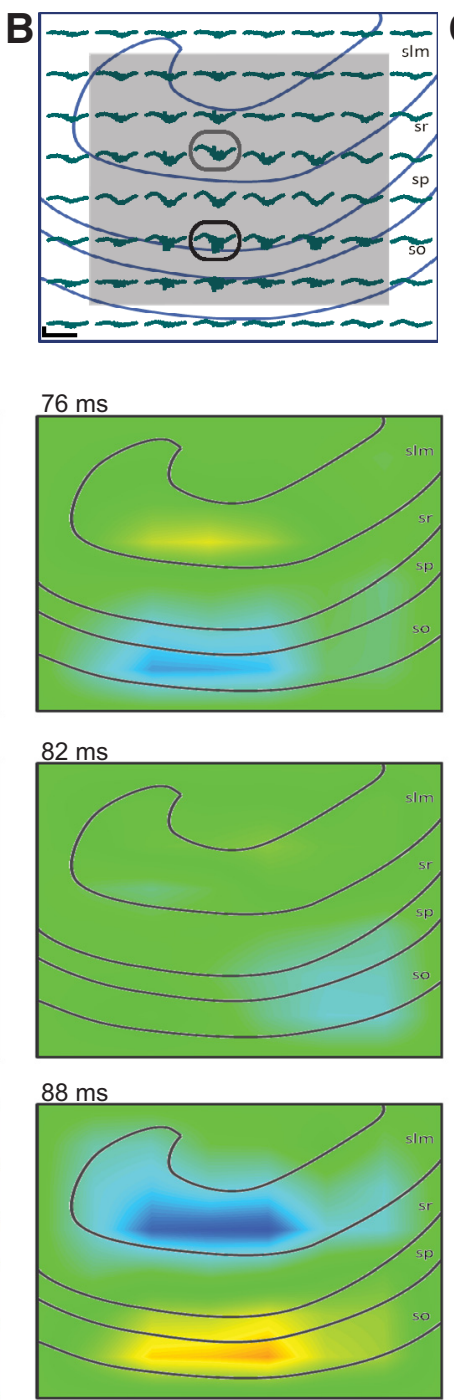
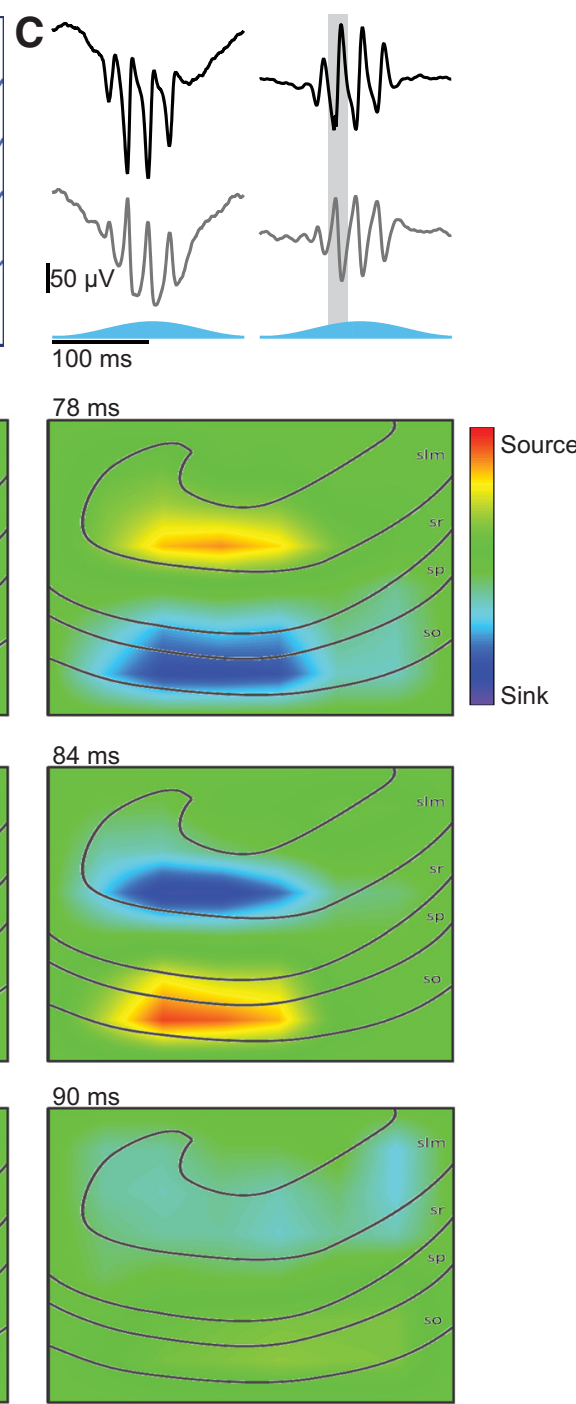

Figure 2. CSD profile of CA1 gamma. A, Location of the multielectrode array used to record CA1 gamma on the hippocampal slice. DG, Dentate gyrus; SUB, subiculum; so, stratum oriens; sp, stratum pyramidale; sr, stratum radiatum; slm, stratum lacunosum-moleculare. $\boldsymbol{B}$, LFP measured from the array shown in $\boldsymbol{A}$. The two channels highlighted with ovals are shown in $\boldsymbol{C}$. Gray shaded area incorporates the electrodes used for the CSD analysis shown in $\boldsymbol{D}$ (outer channels were eliminated to avoid edge effects). Horizontal scale bar, $200 \mathrm{~ms}$; vertical scale bar, $100 \mu \mathrm{V}$. C, Examples of average cycles constructed from all theta bins recorded $(n=48$ ) in the SP (top left, black trace) and SLM (bottom left, gray trace). Data were then bandpass filtered between 30 and $120 \mathrm{~Hz}$ to isolate the gamma oscillations as shown in the right-hand column. The shaded gray area represents the time window used for the CSD shown in $\boldsymbol{D}$. D, 2D CSD profile for the electrodes shown in $\boldsymbol{A}$ at $2 \mathrm{~ms}$ time resolution for the peak gamma cycle. The alternating sink-source pairs were consistent across all slices tested.

To further ascertain that these oscillations were intrinsic to the CA1 and independent of afferent activity, CA1 minislices were prepared from acute hippocampal slices and the oscillations were recorded from both the SP and SLM layers simultaneously (Fig. $3 A$ ). A cut between the border of the CA1 and the subiculum, severing EC-CA1 connections, caused no significant change in the power ( $43 \pm 19 \%$ increase, $p=0.0658, n=18$; Fig. $3 B, C)$ or the frequency $(55 \pm 1 \mathrm{~Hz}$ compared with $58 \pm 4 \mathrm{~Hz}$ before and after the cut respectively, $p=0.4907$; Fig. $3 B, D$ ) of the oscillations recorded from the SP. The oscillations recorded concurrently from the SLM layer also demonstrated no significant change in the power $(6 \pm 13 \%$ increase, $p=0.9698, n=17$; Fig. $3 \mathrm{~B}, C)$ or the frequency $(54 \pm 1 \mathrm{~Hz}$ compared with $55 \pm 1 \mathrm{~Hz}$ before and after the cut respectively, $p=0.1357, n=17$; Fig. $3 B, D)$ when this cut was made. As there is also a gamma generator in the CA3, a second cut was then made along the CA1-CA3 border. Again, this caused no significant change in the power ( $18 \pm 9 \%$ increase, $p=0.4641, n=10$; Fig. $3 B, C)$ or frequency of the oscillations $(56 \pm 1 \mathrm{~Hz}, p=0.6049$; Fig. $3 B, D)$ recorded from the SP. This was also true of the power $(19 \pm 9 \%$ decrease, $p=$ $0.0328, n=15$; Fig. $3 B, D)$ and frequency $(59 \pm 4 \mathrm{~Hz}, p=0.3382$, $n=15$; Fig. $3 C, D)$ of the gamma oscillations recorded from the SLM after the second cut. These gamma oscillations were therefore independent of activity in the afferent regions.

In our transgenic mice, no eYFP expression was observed in CA3 pyramidal neurons, whereas pyramidal neurons in the EC did express eYFP. Accordingly, the EC projections terminating in the SLM could still be activated by light even after the cortex was severed from the hippocampus. Therefore, using a digital micromirror device to focus the light in a narrow band on individual layers of the CA1, we investigated the importance of the different CA1 layers for generating the gamma oscillations (Fig. $3 E$ ). The power of the oscillations was highest when the light was focused over the SP layer, with SO and SR stimulation inducing oscillations with a power of $78 \pm 5 \%$ and $83 \pm 4 \%$ respectively compared with stimulation of the SP $(p=0.0019$ and 0.0055 
A

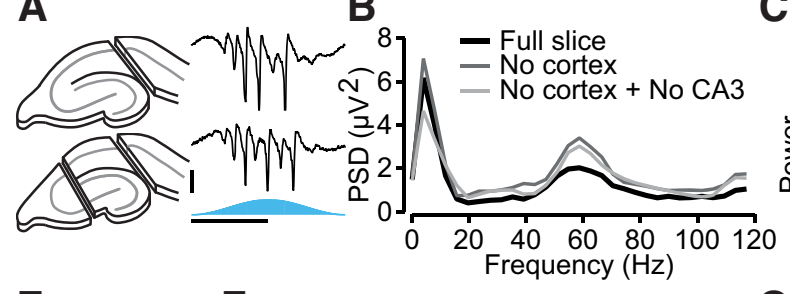

E

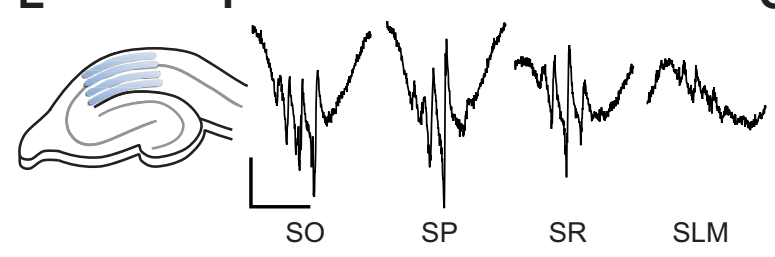

C

G

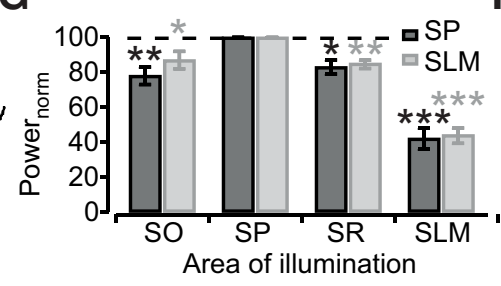

D

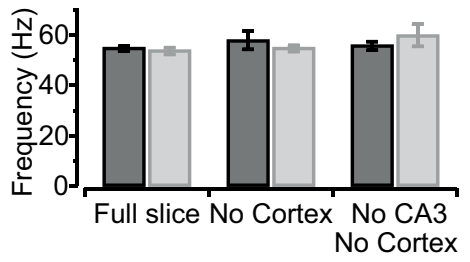

H

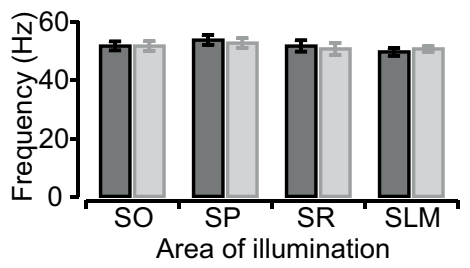

Figure 3. CA1 gamma is independent of upstream regions. $A$, Slices were cut along two axes (left) to isolate the CA1 from the cortex (top row) and from both the cortex and CA3 (bottom row). Right, Representative activity from a single slice recorded from the SP for each condition. The bottom blue trace represents the light stimulation. Vertical scale bar, $50 \mu \mathrm{V}$; horizontal scale bar, 100 ms. $\boldsymbol{B}$, PSD plot for the recordings shown in $\boldsymbol{A}$ and before any cut was made to the slice. C, Average change in the power of the gamma oscillations measured from both the SP (black) and SLM (gray), calculated as the area under the curve $\pm 15 \mathrm{~Hz}$ from the peak in the gamma range $(30-120 \mathrm{~Hz})$, between when slices were intact (left column) to when a cut was made to separate the CA1 from the cortex (middle column, $n=18$ for SP and $n=17$ for SLM), and when the second cut was made to isolate the CA1 from the CA 3 in addition to the cortex (right column: $n=16$ for SP; $n=15$ for SLM). $D$, Same as in $\boldsymbol{C}$ but the peak frequency for the different conditions is plotted. For $\boldsymbol{C}$ and $\boldsymbol{D}$ there are no significant differences when the full slice condition was compared with either of the other two conditions using a paired $t$ test with Bonferroni's corrections ( $p$ value threshold of 0.025 ). $\boldsymbol{E}$, A digital micromirror device was used to precisely deliver light to the slice. This allowed for the light to be concentrated on a thin band spanning a single layer of the CA1 (blue sections). $\boldsymbol{F}$, Representative traces of activity recorded from the SP when the light stimulation was focused in a narrow band on the SO (left), the SP (second from left), the SR (second from right), and SLM (right). Horizontal scale bar, $100 \mathrm{~ms}$; vertical scale bar, $50 \mu \mathrm{V}$. G, Average change in the power of the gamma oscillations measured from both the SP (black) and SLM (gray) calculated in the same manner as in C, depending on the layer that was stimulated with the light. The values are normalized to when the light was focused on the SP layer. $n=14$ for SP and $n=12$ for SLM recordings. ${ }^{*} p<0.0167,{ }^{* *} p<0.0033$, and ${ }^{* * *} p<0.0003$, paired $t$ test with Bonferroni's corrections. $\boldsymbol{H}$, Same as in $\boldsymbol{G}$ but for the average frequency of the oscillations. There were no significant differences in the frequency between SP stimulation and stimulation of any of the other layers when using a paired $t$ test with Bonferroni's corrections ( $p$ value threshold, 0.0167 ). In $\mathbf{C}, \boldsymbol{D}, \boldsymbol{G}$, and $\boldsymbol{H}$, error bars represent SEM.

respectively, $n=14$; Fig. $3 F, G)$. Stimulation of the SLM, the location of EC projections, decreased the oscillations further still to $42 \pm 6 \%(p=0.0001, n=14$; Fig. $3 F, G)$. Furthermore, these changes in power were also observed in the concurrent oscillations recorded from the SLM (SO stimulation: $87 \pm 5 \%$; SR: $85 \pm$ 2\%; SLM: $44 \pm 4 \% ; p=0.0161,0.0008$, and 0.00001 respectively, $n=12$; Fig. $3 G$ ). There was no change in the frequency of the oscillations across all conditions tested (Fig. $3 H$ ). The SP layer was therefore the most important for generation of the gamma oscillations.

Therefore, theta-frequency activation of the CA1 is sufficient to generate gamma oscillations that are intrinsic to the CA1, which we refer to as CA1 gamma. Our next step was to examine the relationship between the theta input and the resulting gamma oscillations that are generated.

\section{CA1 gamma is resilient to changes in the frequency and intensity of theta input}

Using optogenetics to elicit gamma oscillations afforded us temporal control over the input to the CA1. We used this to ask whether different stimulation patterns could influence the properties of the resulting gamma oscillations.

During movement, the frequencies of both hippocampal theta oscillations (McFarland et al., 1975; Sławińska and Kasicki, 1998) and gamma oscillations (Ahmed and Mehta, 2012) increase with the running speed of the animal. Therefore, we asked whether a change in theta frequency influences the resulting gamma oscillations. As stimulation frequency was increased from 1 to $10 \mathrm{~Hz}$, the power of CA1 gamma was highest in the middle of the frequency range, at $5 \mathrm{~Hz}$ stimulation (Fig. $4 A$ ). There was an almost linear decrease either side of this frequency, and the power decreased to $81 \pm 7 \%$ at $1 \mathrm{~Hz}$ stimulation frequency and to $68 \pm$ $11 \%$ at $10 \mathrm{~Hz}$ stimulation frequency (Fig. $4 B ; n=11$ ). Outside of this frequency range, gamma oscillations could still be induced across all stimulation frequencies tested $(0.25-30 \mathrm{~Hz})$, but the power and robustness of the oscillations were dramatically reduced. The frequency of CA1 gamma remained relatively constant throughout the different stimulation frequencies, increasing slightly with stimulation frequency, but by $\leq 10 \mathrm{~Hz}$ (Fig. $4 C)$. Therefore, there is an optimum theta frequency range within which the CA1 oscillates with the strongest gamma power, and this range coincides with the middle of the theta range.

We then investigated the effect of the intensity of theta stimulation on CA1 (Fig. 4D). The power of the gamma oscillations measured from the SP increased with stimulation intensity between 20 and $80 \%$ of maximum light intensity $(1.9-2.1 \mathrm{~mW}$. $\left.\mathrm{mm}^{-2}\right)$, and plateaued between 80 and $100 \%$ intensity $(20 \%$ compared with $80 \%, 24 \pm 4 \%, n=11$; Fig. $4 E$ ). This change in power was mirrored by the change in the amount of inward current in pyramidal neurons elicited by ChR2 across the different light intensities (Fig. 4D,E). Despite these large changes in power, the frequency of the gamma oscillations remained essentially constant across all the different light intensities tested ( $44 \pm$ $1 \mathrm{~Hz}$ at $40 \%$ light intensity compared with $46 \pm 2 \mathrm{~Hz}$ at $100 \%$ intensity; Fig. $4 F$ ).

To analyze the phase locking of the gamma oscillations to the theta stimulation, a CWT was calculated for each stimulation cycle (Fig. 4G). The phase locking of each individual gamma cycle was high within each slice but not between slices, with the gamma in each different slice having slightly different phase preferences relative to the theta stimulation (Fig. $4 H$ ). We then compared the relationship between stimulation intensity and theta-gamma phase-phase coupling by calculating the average $r$ value for the three largest gamma oscillations recorded for each slice under the different stimulation intensities. There was an almost linear increase in the $r$ value as the power of the oscillations increased 
A
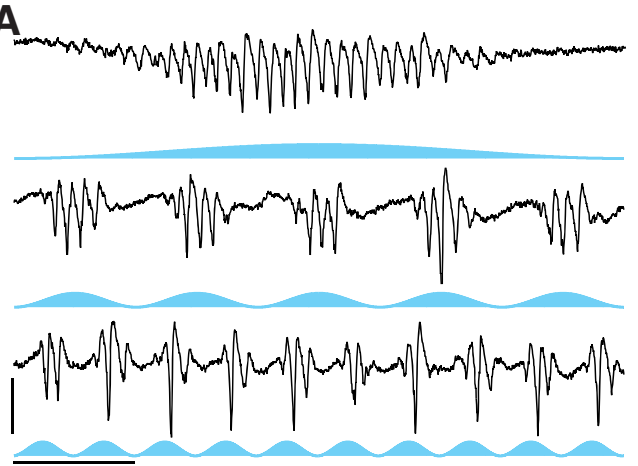

D
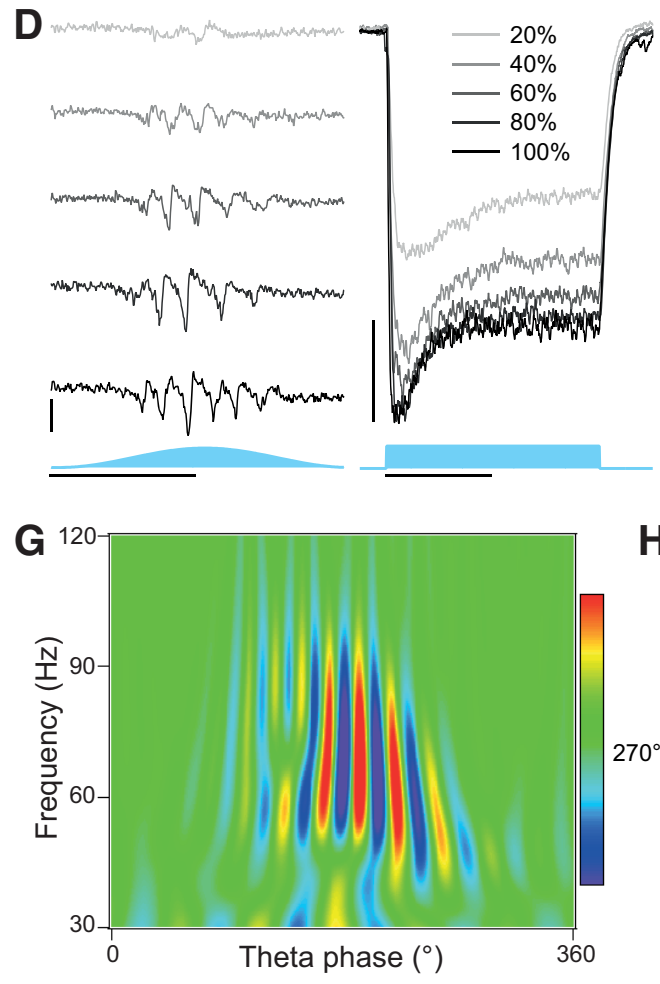

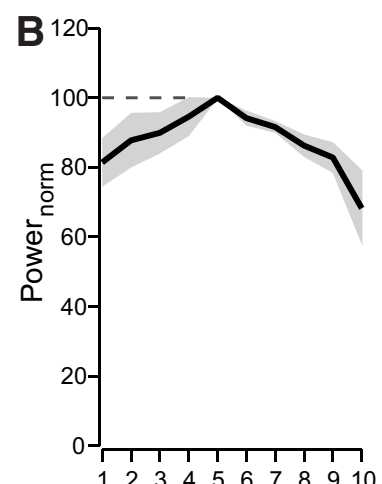

$\begin{array}{llllllllll}1 & 2 & 3 & 4 & 5 & 6 & 7 & 8 & 9 & 10\end{array}$

E
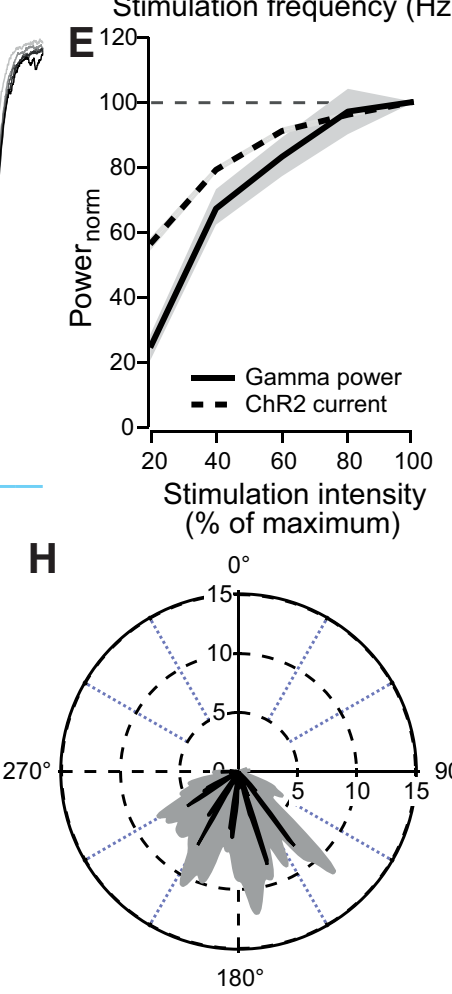

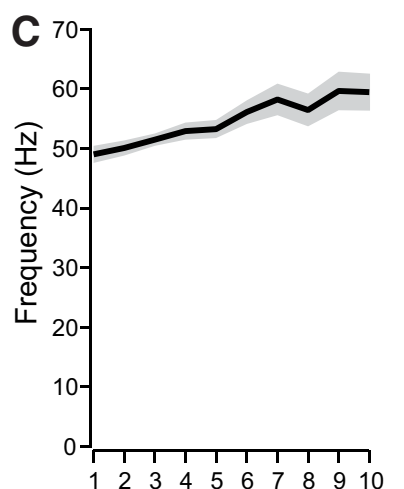

$\mathbf{F}$

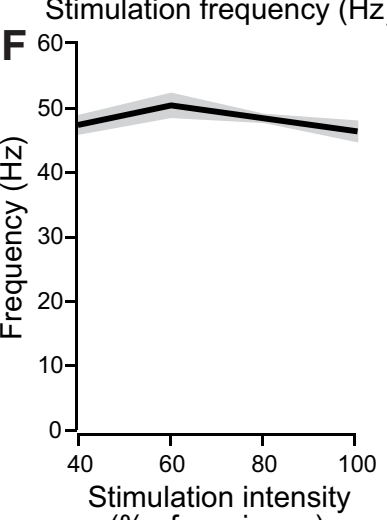

( $\%$ of maximum)

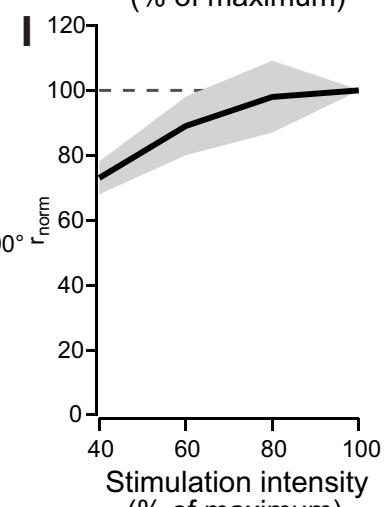

(\% of maximum)

Figure 4. The effect of theta stimulation on CA1 gamma. A, Representative activity recorded from the SP of the CA1 for stimulation at $1 \mathrm{~Hz}$ (top), $5 \mathrm{~Hz}$ (middle), and $10 \mathrm{~Hz}$ (bottom). The shaded blue region represents the optical stimulation of the above trace. $\boldsymbol{B}$, The average change in the power of CA1 gamma measured from the SP as a function of stimulation frequency $(n=10)$. $\boldsymbol{C}$, Same as in $\boldsymbol{B}$ but for the average frequency. $\boldsymbol{D}$, Representative traces of both CA1 gamma (left) and inward current recorded from pyramidal neurons in voltage-clamp mode (right) as stimulation (light) intensity was changed from $20 \%$ of maximum (top) to $100 \%$ of maximum (bottom) in $20 \%$ increments. The shaded blue region represents the optical stimulation. Horizontal scale bar: left, 100 ms; right, $200 \mathrm{~ms}$; vertical scale bar: left, $500 \mu \mathrm{V}$; right, $200 \mathrm{pA}$. $\boldsymbol{E}$, The average change in the power of CA1 gamma measured from the SP as a function of stimulation intensity (solid line, $n=10)$. The average change in inward currents recorded from ChR2 during the different light stimulations is also displayed (dashed line, $n=10$ ). $\boldsymbol{F}$, Same as in $\boldsymbol{E}$ but frequency, rather than power, is displayed. G, Calculating a CWT allows one to see the phase of individual gamma cycles. In this case, the average CWT for all theta bins $(n=30)$ recorded at $100 \%$ light intensity from the single slice shown in $\boldsymbol{A}$ is displayed. $\boldsymbol{H}$, Polar graph histogram of the phase of each individual gamma cycle relative to the theta stimulation. The black trace represents all gamma oscillations recorded from a single example slice ( $n=90$ from 30 theta cycles) and the gray trace represents all gamma oscillations from all slices ( $n=646$ from 8 slices). $I$, The change in $r$ (which represents the strength of the phase coupling between the gamma oscillations and the theta oscillation), normalized to the maximum value, as a function of stimulation intensity. For $B, C, E, F$, and $I$ shaded areas represent $S E M$.

( $27 \pm 5 \%$ increase between 40 and $80 \%$ light intensity; Fig. $4 I$ ), suggesting that theta-gamma phase-phase coupling increases with theta input to the CA1.

To summarize, while the power of CA1 gamma is highly dependent on theta input, the oscillations occupy a specific narrow frequency range that did not shift by more than a few hertz when either the frequency or power of the theta input was varied.

\section{Pharmacological analysis of CA1 gamma}

It was therefore apparent that optogenetic activation of CA1 consistently activated a single internal gamma generator. We next embarked on a pharmacological characterization of this CA1 gamma generator.

When slices were left for an incubation period of $10 \mathrm{~min}$, there was a significant decrease in the power of the oscillations over time by $8 \pm 2 \%(p=0.0078, n=11)$. Therefore, the difference in oscillatory power before and after each drug application was compared with this change. Furthermore, the frequency of CA1 gamma before drug application was found to be different when compared with slices in the control condition, so the change in frequency, rather than the actual values, was used for comparison. Ethanol, DMSO, and $\mathrm{NaOH}$ were used as solvents for some 


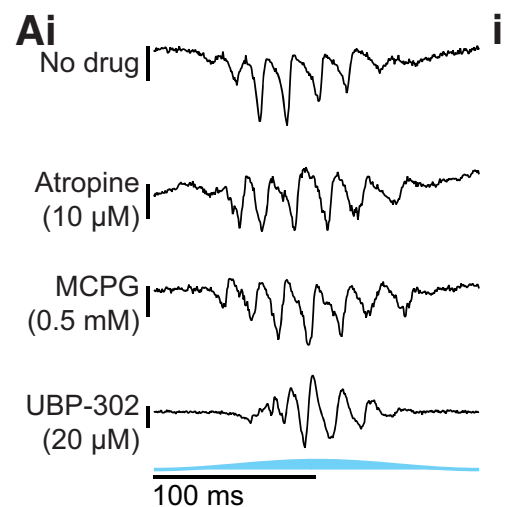

$100 \mathrm{~ms}$
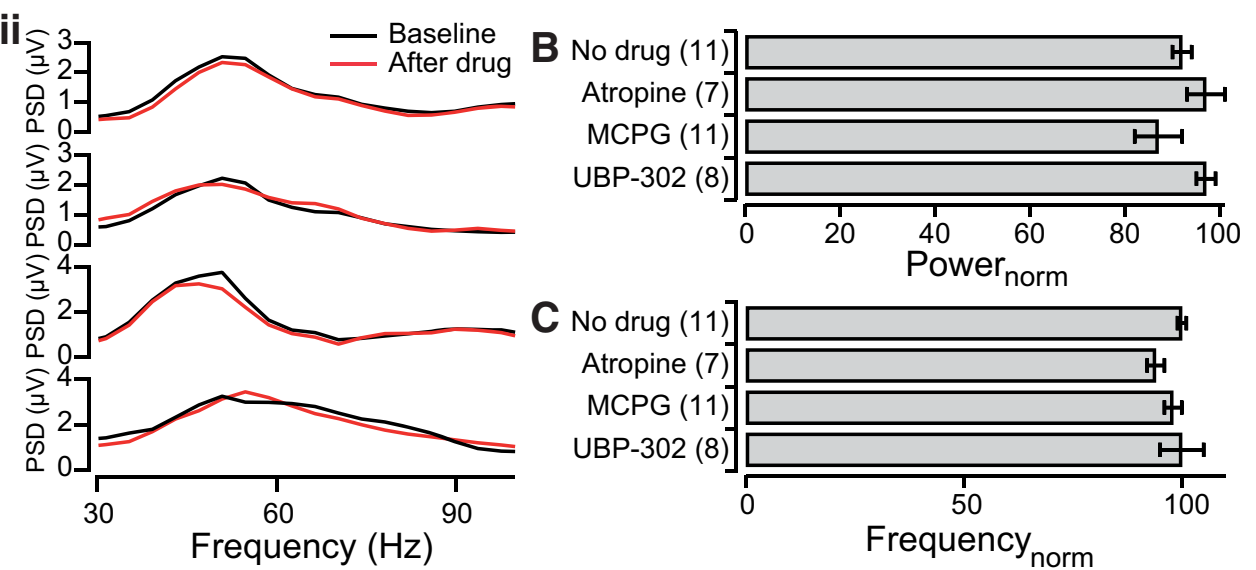

Figure 5. The independence of CA1 gamma from neuromodulators. Ai, Representative traces of extracellular activity recorded in the SP after application of the indicated drug. Numbers in brackets are the concentration of the drug that was used for each condition. Vertical scale bar, $50 \mu$ V. Blue trace represents the optical stimulation. Aii, The PSD of the gamma range for the examples given in $\boldsymbol{A}$ before (black line) and after (red line) drug treatment. $\boldsymbol{B}$, Average change in the power, calculated from the PSD as the area under the curve $\pm 15 \mathrm{~Hz}$ from the peak in the gamma range $(30-100 \mathrm{~Hz})$ for each of the drug conditions. Numbers in parentheses are the $n$ for each condition. The power is normalized to the baseline condition and thus represents percentage change in power 10 min after drug application. Error bars represent SEM. C, Same as in B but the change in the peak PSD frequency in the gamma range, rather than the power, is shown. There were no significant differences in either the power or the frequency between control and any of the drugs tested when assessed using an independent samples $t$ test with Bonferroni's corrections ( $p$ value threshold, 0.005).

of the reagents, and none of these had a significant effect on the power or the frequency of CA1 gamma (data not shown).

We first sought to see whether the CA1 gamma model reported here shares mechanisms with pharmacological models of in vitro gamma oscillations (Fig. 5). Activation of mGlu receptors (mGluRs) causes CA1 gamma oscillations in vitro (Whittington et al., 1995), but mGluRs were not important for optogenetic CA1 gamma, as $500 \mu \mathrm{M}$ of the mGluR antagonist MCPG had no effect on the power $(87 \pm 5 \%, p=0.3618, n=$ 11 ; Fig. $5 B$ ) or frequency of the gamma oscillations ( $98 \pm 2 \%$, $p=0.3115$; Fig. $5 C$ ). Activation of kainate receptors can also induce in vitro CA1 gamma (Fisahn, 1999), but when these receptors were blocked with $20 \mu \mathrm{M} U \mathrm{UBP}-302$, there was no change in the power $(97 \pm 2 \%, p=0.1589, n=8$; Fig. $5 B)$ or frequency of CA1 gamma ( $100 \pm 5 \%, p=0.9923$; Fig. $5 C)$. It was recently shown that muscarinic receptor activity can induce CA1 gamma oscillations (Pietersen et al., 2014), but these receptors were not involved here, as $10 \mu \mathrm{M}$ of the muscarinic receptor antagonist atropine had no effect on the power $(97 \pm$ $4 \%, p=0.2340, n=7$; Fig. $5 B)$ or the frequency $(94 \pm 2 \%, p=$ 0.0222; Fig. 5C) of the CA1 gamma.

Two different models have been proposed for the mechanism of gamma oscillations. The interneuron network gamma (ING) model predicts that recurrently connected interneurons alone are sufficient to generate gamma oscillations, by spiking at their preferred firing rate, which will entrain the entire network (Whittington et al., 1995; Bartos et al., 2007). The second model is the pyramidal-interneuron network gamma (PING) model, whereby pyramidal cell spiking excites interneurons, which then silence pyramidal neurons (as well as other interneurons) via feedback inhibition until the inhibition fades and the pyramidal neurons can fire again, resulting in synchronized rhythmic activity (Fisahn et al., 1998; Whittington et al., 2000). As interneurons are thought to be important for both models, we first tested their involvement in $\mathrm{CA} 1$ gamma by blocking $\mathrm{GABA}_{\mathrm{A}}$ receptors with $10 \mu \mathrm{M}$ bicuculline (Fig. 6). This blocked the gamma oscillations (32 $\pm 4 \%, p=0.00001, n=9$; Fig. $6 B)$. Thiopental $(20 \mu \mathrm{M})$, which prolongs IPSCs, showed a trend toward decreasing the frequency, though this was not significant $(4 \pm 3 \%$ from $52 \pm 1$
$\mathrm{Hz}$ to $49 \pm 2 \mathrm{~Hz}, p=0.03, n=8$; Fig. $6 C)$. This was accompanied by a significant decrease in the power of the oscillations $(74 \pm$ $3 \%, p<0.0001$; Fig. $6 B$ ).

Next, we investigated the importance of excitation for CA1 gamma by blocking glutamatergic excitation using $50 \mu \mathrm{M}$ APV and $20 \mu \mathrm{M}$ NBQX to block AMPA, kainate, and NMDA receptors (Fig. 7). This caused a significant reduction in the power of the oscillations to $37 \pm 5 \%(p<0.0001, n=9$; Fig. $7 B)$. Using $50 \mu \mathrm{M}$ APV alone had no effect on the power ( $96 \pm 6 \%, p=0.6194, n=$ 13; Fig. $7 B$ ) or frequency ( $96 \pm 2 \%, p=0.0749$; Fig. $7 C$ ) of the gamma oscillations, suggesting that NMDA receptors have no role in optogenetically induced CA1 gamma oscillations. Conversely, when AMPA and kainate receptors were blocked with 50 $\mu \mathrm{M}$ NBQX, CA1 gamma was blocked (power reduced to $35 \pm$ $3 \%, p<0.0001, n=14$; Fig. $7 B$ ). Twenty micromolar GYKI53655 was then used to identify which of these two excitatory receptors was important. At this concentration, GYKI-53655 blocks $\sim 80 \%$ of AMPA receptors while not affecting kainate receptors (Paternain et al., 1995), and this also significantly reduced the power of the oscillations $(68 \pm 7 \%, p=0.0002, n=9$; Fig. $7 B)$, whereas the specific kainate receptor antagonist UBP-302 had no effect ( $97 \pm 2 \%, p=0.1589, n=8$; Fig. $5 B$ ).

None of the aforementioned excitatory blockers altered the frequency of the gamma oscillations produced (Fig. 7C). Even CTZ, which prolongs EPSCs, had no effect on the power (85 \pm $4 \%, p=0.0676$; Fig. $7 B$ ) or the frequency of the oscillations $(108 \pm 5 \%, p=0.0725$; Fig. $7 C)$.

We conclude that for CA1 gamma to be generated, both $\mathrm{GABA}_{\mathrm{A}}$ receptor-mediated inhibition and AMPA receptormediated excitation are required. These results suggest that the PING model underpins CA1 gamma. We then sought to confirm this conclusion by conducting single-cell recordings during CA1 gamma.

\section{Single-cell recordings}

To understand the underlying mechanisms at the cellular level during CA1 gamma, patch-clamp recordings were undertaken from individual CA1 neurons during optical theta stimulation, while theta and gamma oscillations were monitored with an ex- 

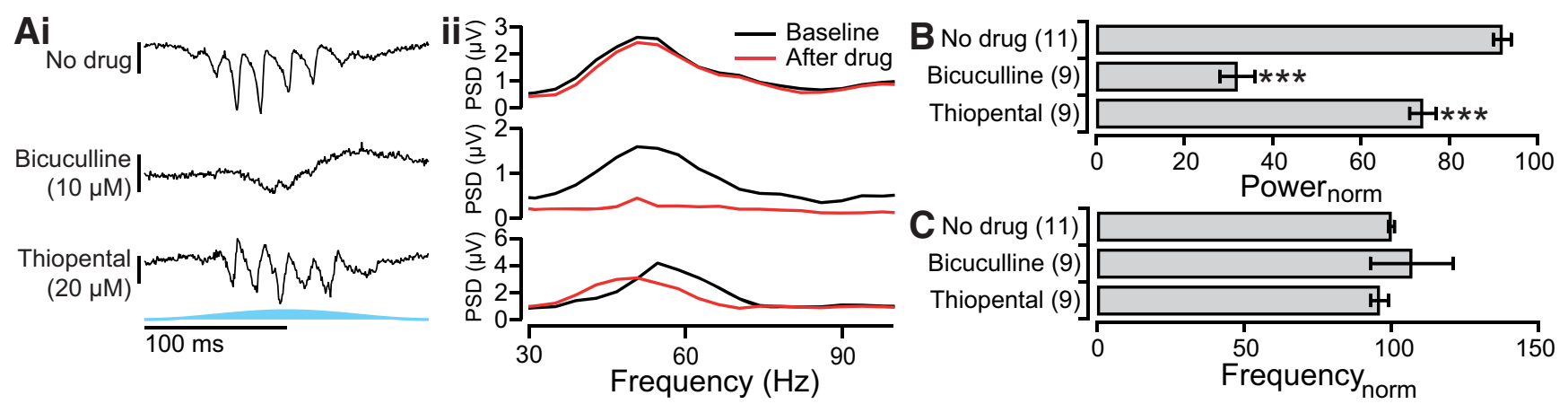

Figure 6. The importance of GABAergic inhibition for CA1 gamma. Ai, Representative traces of extracellular activity recorded in the SP after application of the indicated drug. Numbers in parentheses are the concentration of the drug that was used for each condition. Vertical scale bar, $50 \mu V$. The blue trace represents the optical stimulation. Aii, The PSD of the gamma range for the examples given in $\boldsymbol{A}$ before (black line) and after (red line) drug treatment. $\boldsymbol{B}$, Average change in power, calculated from the PSD as the area under the curve $\pm 15 \mathrm{~Hz}$ from the peak in the gamma range $(30-100 \mathrm{~Hz})$ for each of the drug conditions. Numbers in parentheses are the $n$ for each condition. The power is normalized to the baseline condition and thus represents percentage change in power 10 min after drug application. Error bars represent SEM. ${ }^{* * *} p<0.0001$, independent samples $t$ test with Bonferroni's corrections. $\boldsymbol{C}$, Same as in $\boldsymbol{B}$ but the change in the peak PSD frequency in the gamma range, rather than the power, is shown. There were no significant differences in the change in frequency between control and any of the drugs tested when assessed using an independent samples $t$ test with Bonferroni's corrections ( $p$ value threshold, 0.005).
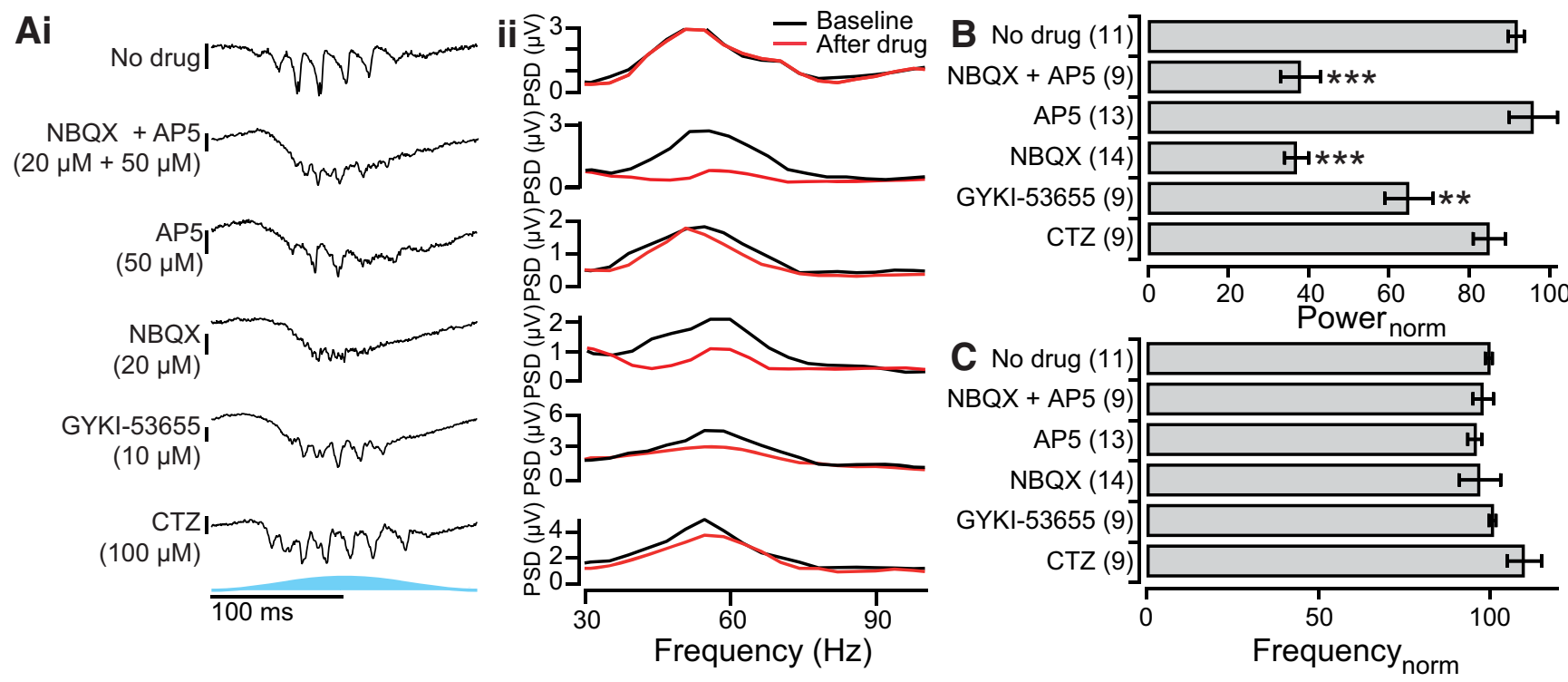

Figure 7. The importance of excitation for CA1 gamma. Ai, Representative traces of extracellular activity recorded in the SP after application of the indicated drug. Numbers in parentheses are the concentration of the drug used for each condition. Vertical scale bar, $50 \mu \mathrm{V}$. The blue trace represents the optical stimulation. Aii, The PSD of the gamma range for the examples given in $A$ before (black line) and after (red line) drug treatment. $\boldsymbol{B}$, Average change in power, calculated from the PSD as the area under the curve $\pm 15 \mathrm{~Hz}$ from the peak in the gamma range $(30-100 \mathrm{~Hz}$ ) for each of the drug conditions. Numbers in brackets are the $n$ for each condition. The power is normalized to the baseline condition and thus represents percentage change in power 10 min after drug application. Error bars represent SEM. C, Same as in B but the change in the peak PSD frequency in the gamma range, rather than the power, is shown. ${ }^{* *} p<0.001$, ${ }^{* * *} p<0.0001$, independent samples $t$ test with Bonferroni's corrections.

tracellular electrode placed in the SP layer of the slice. Cells were filled with neurobiotin and reconstructed post hoc to confirm the cell type ( $n=50$ of 62 ), which allowed for identification of pyramidal neurons, with their distinctive main apical dendrite, and the smaller, more compact, interneurons (Fig. 8A). All reconstructed interneurons resided in deep SP and SO and possessed a range of morphologies (Fig. 8A). The firing properties of all cells from which recordings were made were also analyzed. While some interneurons display an irregular spiking pattern $(n=2$ of 18), most displayed a fast-spiking pattern (stuttering, $n=2$ of 18 ; continuous, $n=14$ of 18 ). The latter, exhibiting consistently narrow action potentials with strong afterhyperpolarization, is widely regarded to be important for gamma generation (Buzsáki and Wang, 2012). In contrast, all pyramidal neurons showed action potential broadening and spike frequency adaptation (Fig. $8 B$ ).
Cell-attached recordings showed that both pyramidal neurons (Fig. 8Ci) and interneurons (Fig. 8Cii) increased their spiking activity in response to blue light, demonstrating the effect of ChR2 on the cells. To distinguish between direct activation of cells expressing ChR2 and indirect activation via synaptic excitation from upstream ChR2-expressing neurons, we recorded from cells in the presence of $50 \mu \mathrm{M}$ AP5 and $20 \mu \mathrm{M}$ NBQX to block all glutamatergic excitation in CA1. This would stop indirect activation occurring but still allow for direct activation. This block had no effect on pyramidal neurons, with all 11 cells recorded in the presence of the blockers spiking in response to light activation in the same manner as cells without blockers (Fig. 8Di). In contrast, of the 11 interneurons recorded from in the presence of the blockers, 10 did not spike in response to light in the presence of the excitatory blockers (Fig. 8Dii) and only one continued to show a strong response to the light stimulation. It was still possi- 
A
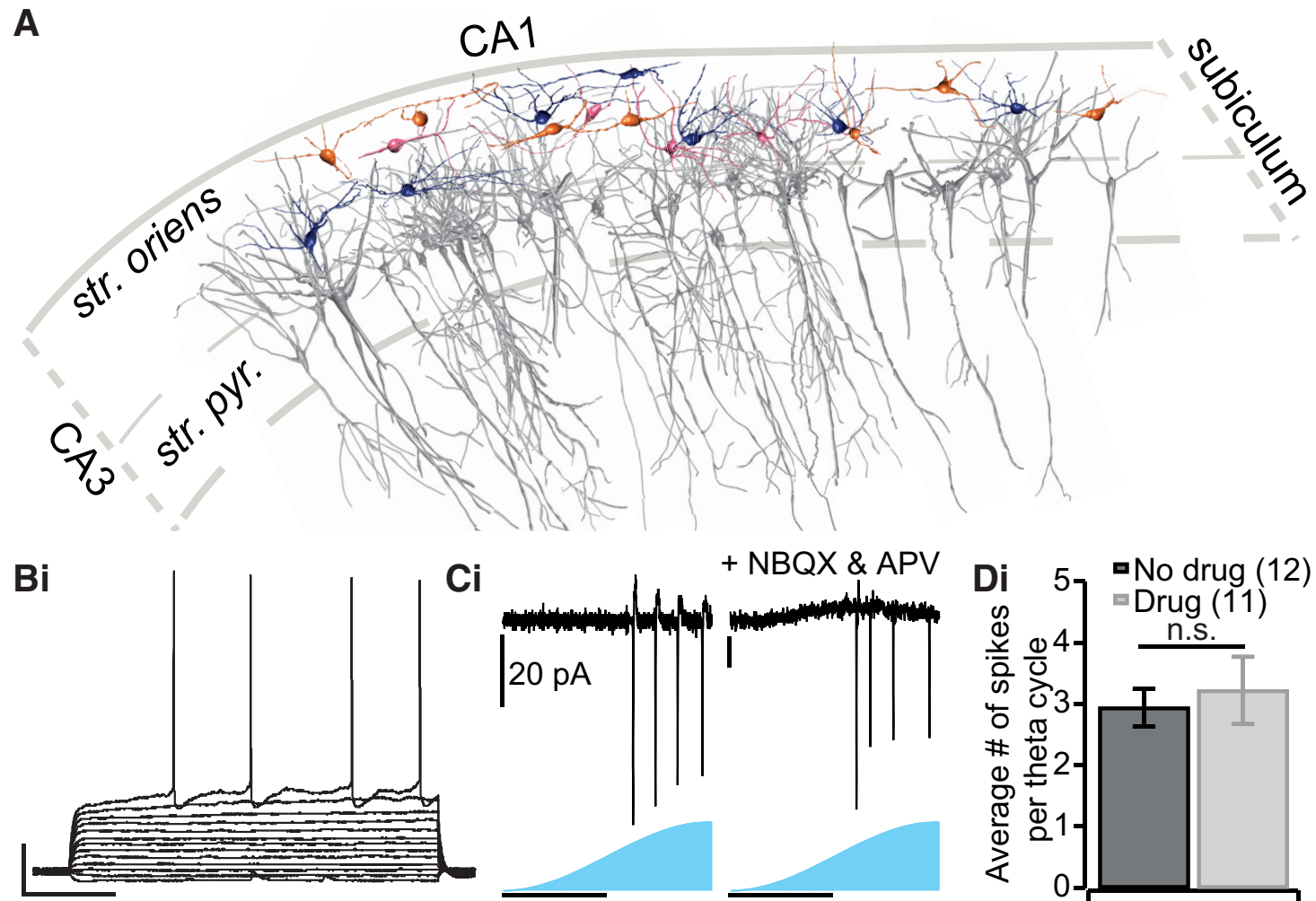

Ci
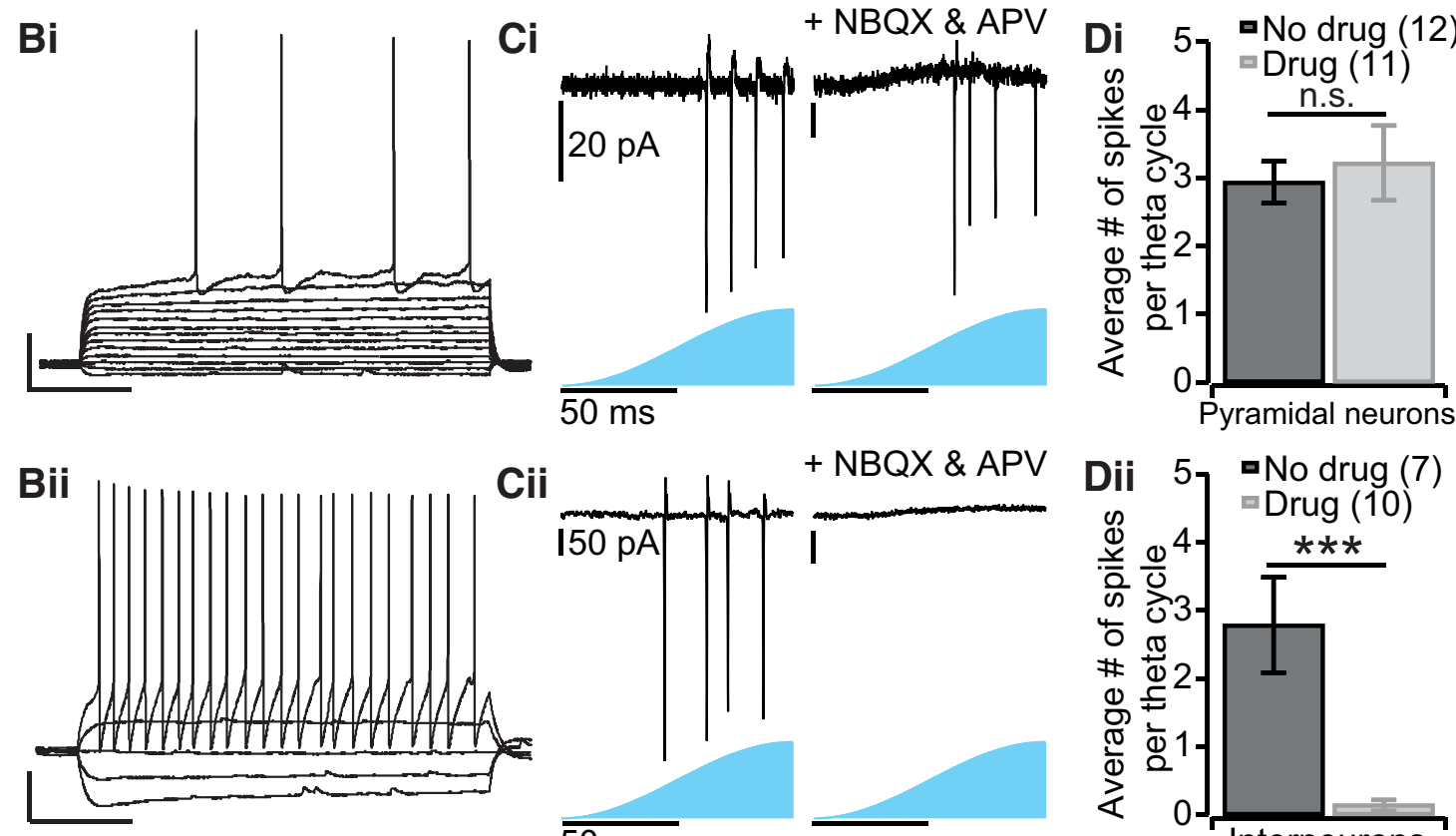

Cii
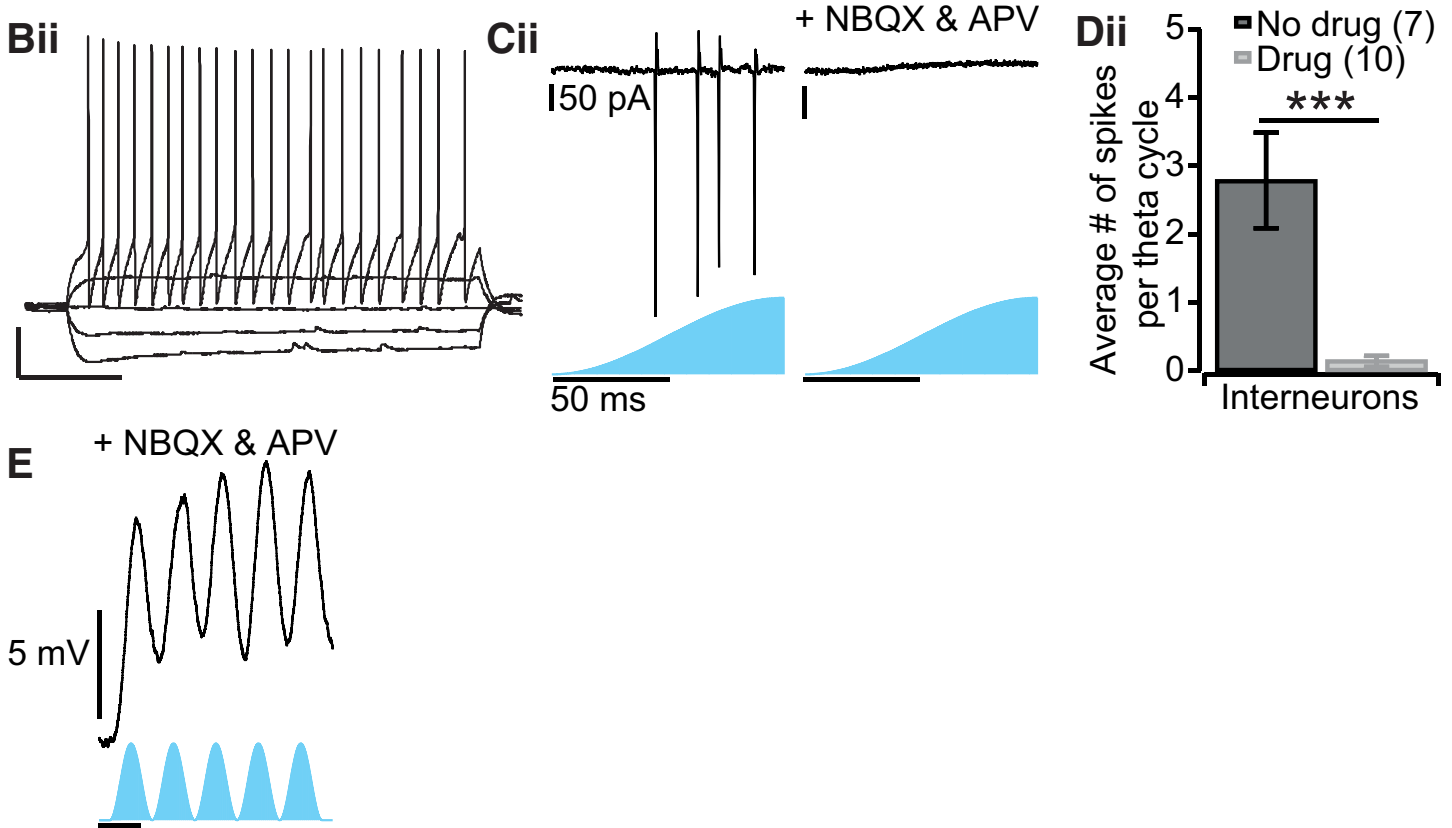

$\overline{200} \mathrm{~ms}$

Figure 8. Characterization and ChR2 expression of CA1 neurons. A, 3D reconstruction of the pyramidal neurons (gray, 32 reconstructed of 36 included) and the interneurons used for the pharmacology (blue, 4 of 11), cell-attached (orange, 7 of 7), and voltage-clamp (pink, 7 of 8) single-cell experiments. The highly distinct morphologies and positioning of the two cell types is obvious. In this schematic CA1 illustration, the relative position and size of the cells were based on the images acquired in the confocal microscope. $\boldsymbol{B}$, The typical membrane potential response observed in response to current steps of increasing amplitude in pyramidal cells (Bi) and fast-spiking interneurons (Bii). $(\boldsymbol{C}$, Typical response of pyramidal neurons ( $\boldsymbol{C} \boldsymbol{i}$ ) and fast-spiking interneurons (Cii) to optical stimulation with (right) and without (left) the presence of the glutamatergic blockers NBQX (20 $\mu \mathrm{M})$ and AP5 (50 $\mu \mathrm{M})$. Di, Average number of spikes that occurred per theta stimulation cycle for pyramidal neurons in control and in pyramidal neurons in the presence of NBQX and AP5. Dii, Average number of spikes that occurred per theta stimulation cycle for interneurons in control and interneurons in the presence of NBQX and AP5. One interneuron retained its response to light in the presence of excitatory blockers, indicating that it expressed ChR2. This interneuron was not included in the group average in Dii. ${ }^{* *} p<0.0005$, independent samples $t$ test with Bonferroni's corrections. n.s. Not significant. Error bars represent SEM. $\boldsymbol{E}$, An example of the typical response of interneurons in response to blue-light stimulation in the presence of excitatory blockers. Light stimulation still caused a slight membrane depolarization, indicating there was some low-level ChR2 expression. 

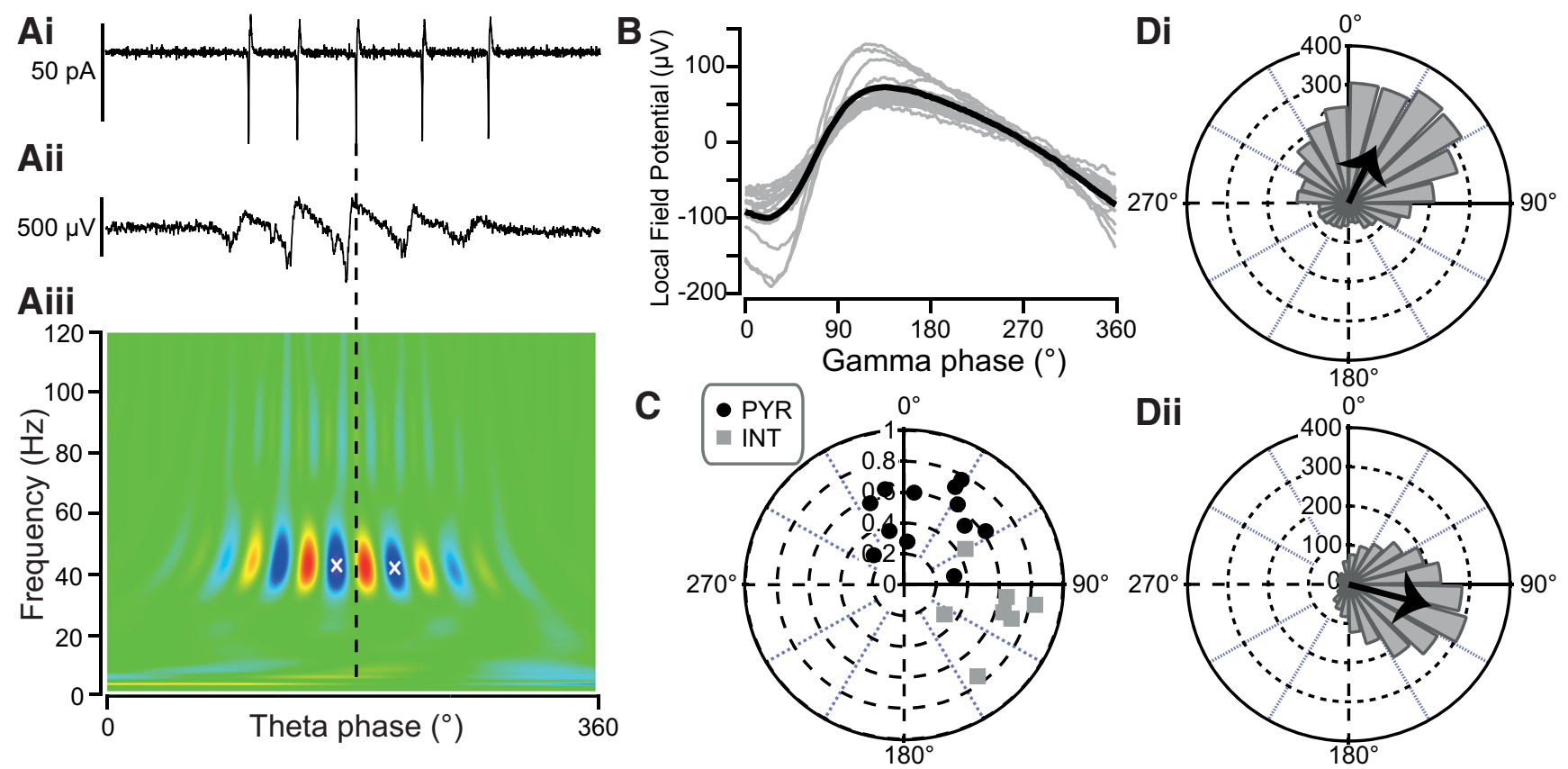

Figure 9. Cell-attached recordings during optogenetic CA1 gamma oscillations. Ai, Spiking activity recorded using cell-attached patch of an example interneuron in CA1 gamma. Aii, The concurrent LFP of the CA1 using an extracellular recording electrode placed in the SP of the CA1. Aiii, CWT of the LFP shown in Aii. The two white crosses mark the location of the adjacent troughs of the gamma oscillation flanking the selected action potential shown in $\boldsymbol{A i}$ (dashed line) and were used to define $0^{\circ}$ and $360^{\circ}$ in the calculation of the phase of the selected action potential relative to CA1 gamma. $\boldsymbol{B}$, The average gamma cycle recorded from each individual slice (gray lines) and the average across all data used in the cell-attached experiments (black line, $n=6443$ from 19 slices). C, The mean angle and $r$ value for all pyramidal neurons (black circles) and interneurons (gray squares) from which recordings were made. $\boldsymbol{D}$, Polar histogram plots (rose plots) to display the phase at which all spikes occurred relative to CA1 gamma for pyramidal neurons (Di; $n=3766$ from 12 slices) and interneurons (Dii; $n=2680$ from 7 slices). The black arrows are the circular mean and accompanying $r$ value for each group (the axis for these arrows is between 0 and 1 at the bottom and top of the histogram respectively).

ble, however, that there was low ChR2 expression in interneurons that could depolarize the cells, just not enough to initiate action potential firing. So the interneurons were also repatched and recordings from them were made in whole-cell current-clamp mode during light stimulation (Fig. 8E). The blue light caused depolarization of the interneurons by an average of $10.0 \pm 1.2$ $\mathrm{mV}(n=10)$. Thus, ChR2 was expressed at high levels in all pyramidal neurons and to a small degree in interneurons, directly triggering spiking in only a small percentage of interneurons $(<10 \%)$.

We then examined the relationship between single-cell events and CA1 gamma. We first used cell-attached patch clamp as a minimally invasive method of recording action potentials from the CA1 neurons. To define the phases of the concurrent gamma oscillation, a CWT was constructed for the theta cycle and the flanking $50 \mathrm{~ms}$ of activity either side. This was then used to identify the time points of the trough of the cycles before and after each event by using the negative peak in the gamma range (30$120 \mathrm{~Hz}$ ) from the CWT. These two adjacent CWT peaks were then used to define $0^{\circ}$ and $360^{\circ}$ respectively (Fig. $9 A$ ). The gamma cycle waveforms were consistent between the different slices, with the trough of the gamma waveform occurring at a mean phase angle of $18^{\circ}(r=0.99, n=19$ cells; Fig. $9 B)$ and the peak occurring at $139^{\circ}(r=0.97, n=19$ cells; Fig. $9 B)$ relative to the troughs extracted from the CWT.

Recordings were made from 12 pyramidal neurons and 7 interneurons. Using Rayleigh's test for uniformity, all of these pyramidal neurons and interneurons were found to have significantly nonuniform firing patterns, with only one of the pyramidal neurons having a $p$ value $>0.0001(p=0.0081)$. All interneurons and pyramidal neurons could be clearly segregated, depending on the phase of their firing relative to CA1 gamma
(Fig. 9C). For pyramidal neurons, the mean angle across all spikes was $18^{\circ}(r=0.45, n=3724$; Fig. 9Di). Interneuron spiking occurred later, with a mean angle across all spikes of $105^{\circ}(r=0.53$, $n=2688$; Fig. 9Dii). Therefore, when action potentials were measured in cell-attached mode, pyramidal neuron firing occurred at the trough of the gamma cycle and interneuron firing occurred a quarter of a gamma cycle later during the ascending phase.

Once we had elucidated the cells' output during CA1 gamma, we next turned to their inputs. Recording in voltage-clamp mode and clamping the cell at $-10 \mathrm{mV}$ (the reversal potential of EPSCs) and $-70 \mathrm{mV}$ (the reversal potential of IPSCs) enabled us to measure respectively inhibitory and excitatory inputs into the cells during CA1 gamma (Fig. 10A). The IPSC input into all pyramidal neurons tested was significantly nonuniform $(p<$ 0.001 in all cases). Of the eight interneurons tested, all demonstrated IPSCs and EPSCs during CA1 gamma apart from one interneuron that received no detectable IPSCs, and one interneuron that received no detectable EPSCs, and were therefore excluded from further analysis. The phase of IPSC input into the seven interneurons was all strongly nonuniform with respect to CA1 gamma (all $p<0.001$ apart from one cell that had $p=$ 0.001). Likewise, the EPSCs into each of the seven interneurons were found to be significantly nonuniform $(p<0.001$ in all cases).

IPSCs occurred at the peak of the gamma cycle, and shared the same phase preference regardless of whether they were recorded in interneurons (mean angle, $201^{\circ} ; r=0.58 ; n=2178$ ) or pyramidal neurons (mean angle, $181^{\circ} ; r=0.56 ; n=2407$; Fig. 10B). EPSCs in interneurons were strongly phase-locked to the ascending phase of CA1 gamma (mean angle, $82^{\circ} ; r=0.5 ; n=1483$ ). This was consistent across all cells, apart from the EPSCs in one 
A

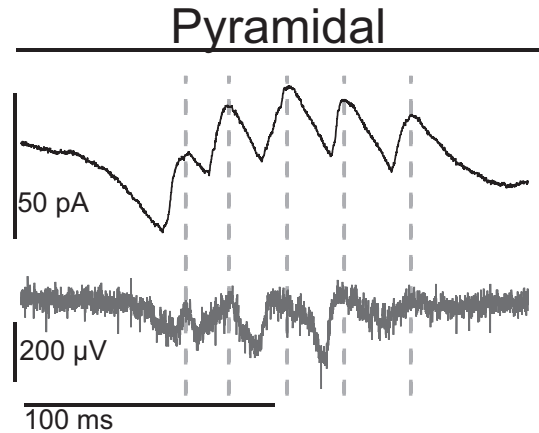

Interneuron
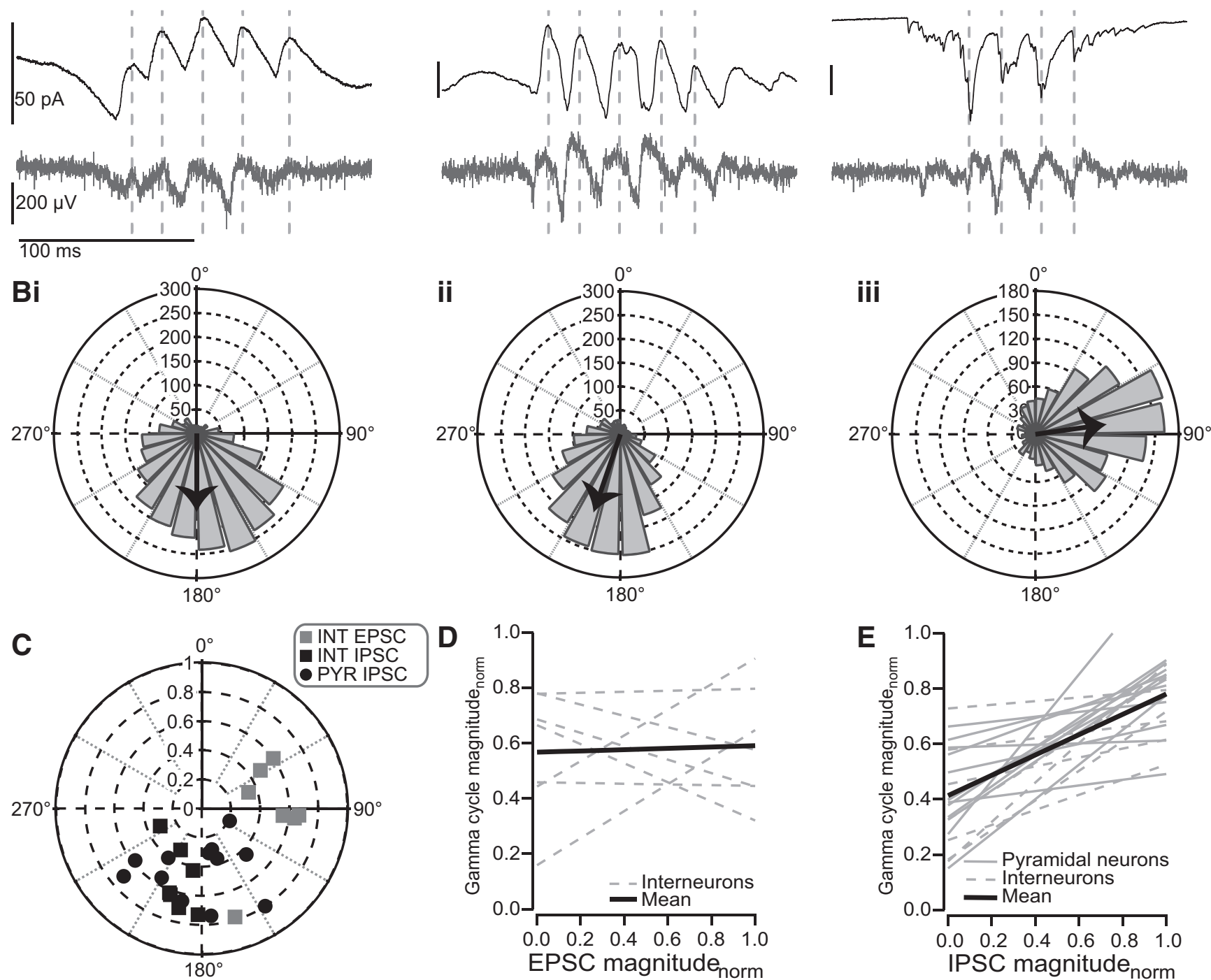

Figure 10. Voltage-clamp recordings during optogenetic CA1 gamma oscillations. $\boldsymbol{A}$, Representative traces of intracellular currents (top) recorded from pyramidal neurons (left) and interneurons (center, right) while the cells were held at $-10 \mathrm{mV}$ (left, center) and $-70 \mathrm{mV}$ (right) to record IPSCs and EPSCs respectively. The concurrent LFP is displayed below each recording. $\boldsymbol{B}$, Polar histogram plot (rose plot) to display the phase at which all recorded IPSCs in pyramidal neurons (Bi; $n=2111$ from 13 cells) and interneurons (Bii; $n=2179$ from 7 cells), and EPSCs in interneurons (Biii; $n=1440$ from 7 cells) occurred relative to CA1 gamma. The black arrows are the circular mean and accompanying $r$ value for the group (the axis for these arrows is between 0 and 1 at the bottom and top of the histogram respectively). $C$, Mean angle and $r$ value for each cell for all the different conditions. D, EPSCs and the size of the concurrent gamma oscillation were normalized to their maximum and linear regression used to calculate a line of best fit for the data from each cell (gray traces) and the average across all cells (black trace; $n=7$ ). $\boldsymbol{E}$, The same as in $\boldsymbol{D}$ but for IPSCs recorded from both pyramidal neurons (gray solid lines; $n=13$ ) and interneurons (gray dashed line; $n=7$ ) and the average regression across all cells from which recordings were made (black line; $n=20$ ).

interneuron, which had a mean angle of $163^{\circ}(r=0.78, n=76$; Fig. $10 C)$. There was therefore a delay between excitatory and inhibitory events that matched the delay between spiking of pyramidal neurons and interneurons.

To assess the contribution of excitatory and inhibitory events to the signal recorded in the LFP, we compared the fluctuations in PSC amplitude to fluctuations in the magnitude of the simultaneously recorded gamma cycle. While there was no correlation between EPSC magnitude and the concurrent gamma cycle magnitude (Fig. $10 D)$, there was a strong correlation between IPSC magnitude and gamma cycle magnitude, which was present for the IPSCs recorded from both pyramidal neurons and interneurons (Fig.
$10 E)$. This therefore reinforces the theory that LFP signals comprise mainly inhibitory events (Oren et al., 2010).

These single-cell results suggest the following mechanism by which CA1 is able to intrinsically generate gamma oscillations (Fig. 11). During each gamma cycle (Fig. 11A), pyramidal neurons first fire action potentials at the start of the cycle (Fig. 11B). The resulting EPSCs then arrive in interneurons (Fig. 11C), causing them to produce action potentials (Fig. 11D). This then results in IPSCs reaching both pyramidal neurons and interneurons, resulting in their inhibition (Fig. 11E) and a silent CA1 until the start of the next gamma cycle. This activity will then persist for as long as there is sufficient theta input into the CA1 to maintain 


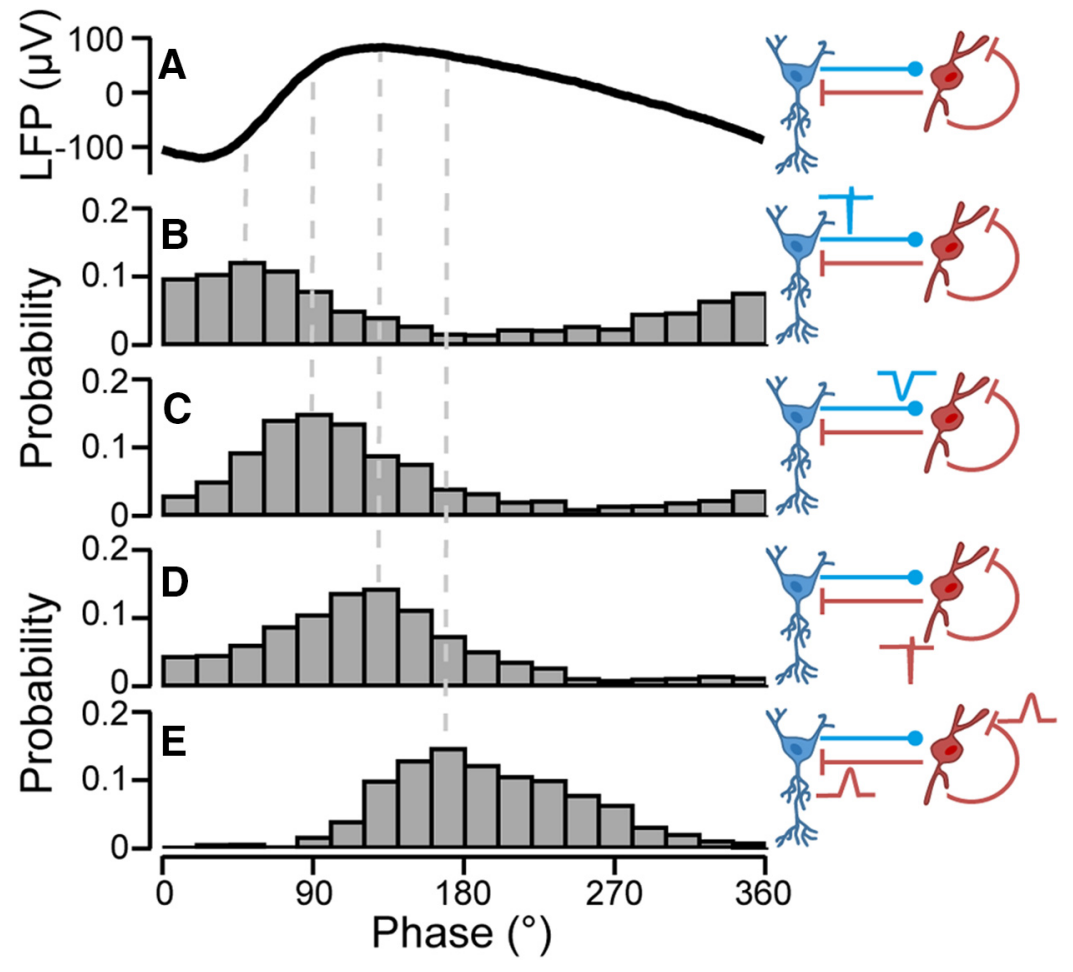

Figure 11. Summary of cellular events during CA1 gamma. $\boldsymbol{A}-\boldsymbol{E}$, The timing of the average gamma cycle $(\boldsymbol{A})$ compared with the probability of the occurrence of pyramidal cell spiking $(\boldsymbol{B})$, EPSCs reaching interneurons $(\boldsymbol{C})$, interneuron spiking $(\boldsymbol{D})$, and IPSCS reaching pyramidal neurons $(\boldsymbol{E})$. Depicted on the right is a schematic of the connections in the CA1 between pyramidal neurons (blue) and interneurons (red) and the location of each step in the circuit.

pyramidal neuron spiking (as evidenced by our experiment modulating the frequency of the theta stimulation).

\section{Discussion}

Our three main findings are as follows: (1) optogenetic stimulation of the CA1 activates a single intrinsic gamma generator; (2) the induced oscillation is robust and largely insensitive to changes in the frequency and power of theta input; and (3) CA1 gamma is generated by a PING mechanism.

\section{Optogenetic theta stimulation of the CA1 induces a single intrinsic gamma oscillation}

Expression of ChR2 in the CA1 allowed for robust induction of gamma oscillations in response to theta stimulation. There are $\geq 3$ distinct gamma oscillations in CA1 in vivo, distinguished by their anatomical site of generation and classified by their frequency ranges as slow $(30-80 \mathrm{~Hz})$, medium $(60-120$ $\mathrm{Hz}$ ), and fast gamma (>100 Hz; Schomburg et al., 2014). Recent in vivo recordings found the CA1 not to be entrained to the slow or medium gamma from its afferent regions, but rather CA1 pyramidal neurons were phase-locked to fast gamma, suggesting an intrinsic CA1 gamma oscillation generator (Schomburg et al., 2014). We provide further evidence for an intrinsic CA1 gamma generator here by demonstrating that optogenetic theta stimulation of the CA1 causes a single gamma oscillation of a narrow frequency range that is independent of activity in the afferent regions. This in vitro CA1 gamma has several similarities with CA1 gamma in vivo, including a relatively fast frequency (Belluscio et al., 2012), similar phase-locking of CA1 gamma to the concurrent theta oscillation (Schomburg et al., 2014) and a similar phase delay between pyramidal neuron firing and interneuron firing within each gamma cycle (Senior et al., 2008; Lasztóczi and Klausberger, 2014).

The presence of the alternating sinksource pair within the CA1 shows that the current generator is within the CA1 itself, as reflected by the reversal of the LFP between the SP and SLM. The gamma oscillations could also be recorded from the neighboring dentate gyrus and subiculum, but as these oscillations were of much smaller amplitude and as no phase reversal was observed in these regions, it is likely that this was due to volume conduction from the CA1, rather than due to the activity of any local generator. Indeed, when the light was directed over these neighboring areas, local gamma oscillations with characteristics distinct from those of CA1 gamma were observed, and in this case phase reversal was detected between the different local layers (data not shown). This highlights the potential of the model presented here for comparing gamma oscillations from different regions of the mouse brain in vitro. The ability of the CA1 to generate its own gamma oscillation independent of afferent regions makes it unlikely that gamma oscillations are an interregionally synchronized process, with each packet of information bound to a specific gamma cycle, which carries it along the different steps of the hippocampal circuit. Instead it seems more likely that gamma oscillations are for unidirectional signal propagation, producing local synchrony and delivering each packet to the next downstream region, whereupon a new gamma oscillation from the local generator takes over transmission of the signal. Indeed, the differences in frequencies between the hippocampal gamma oscillations demonstrate that the gamma generators do not need to align their frequency ranges for successful cognitive processing. Having different frequencies would result in asynchrony between the different signals. Perhaps this even enhances processing capability by allowing each area to select input signals by the gamma frequency that carries those signals.

\section{Theta input has only a small effect on CA1 gamma}

CA1 gamma was remarkably robust to changes in the frequency and power of theta stimulation (Fig. 4). When the frequency of the theta stimulation was altered, the gamma power was highest for the middle of the theta range. Such theta resonance also exists in vivo (Stark et al., 2013), further supporting the physiological relevance of this model of intrinsic CA1 gamma. When we manipulated the strength of the theta input, the power of the gamma oscillation increased as theta stimulation intensity was increased. Interestingly, both theta power (Chen et al., 2011) and gamma power $>60 \mathrm{~Hz}$ (Ahmed and Mehta, 2012) was found to increase with running speed in vivo. Therefore, perhaps the increase in gamma power is a simply a consequence of the increase in theta power, rather than being directly caused by the increase in running speed.

Despite dramatically varying both the intensity and the frequency of the theta stimulation, the frequency of the CA1 gamma never strayed by more than a few hertz from the original fre- 
quency, indicating that it is a rigid phenomenon. As the frequency for intrinsic CA1 gamma was far higher than for slow gamma as seen in vivo (as well as the cholinergically induced slow gamma in vitro; Fisahn et al., 1998), CA3 activity might be required for slow gamma in CA1 (Csicsvari et al., 2003; Colgin et al., 2009). This finding supports the theory that CA3 interneurons and CA3-CA1 feedforward inhibition are important for CA1 slow gamma (Zemankovics et al., 2013). It is likely that, in terms of gamma generation in the CA1, there are $\geq 2$ different circuits consisting of interneuron populations with different inhibitory time constants (which would set the different oscillation frequencies), and in the model presented here, predominantly one such population is being activated. All interneurons tested here were found to participate in CA1 gamma and all had similar phase preferences. Our sampling of interneurons was limited to fast-spiking cells in the deep SP and SO. Therefore it is possible that targeting other interneuron populations may uncover some possessing a different relationship with CA1 gamma.

It has been assumed that one role of gamma oscillations is to synchronize the timing between networks, thus allowing for successful neural communication by providing a rigid temporal framework (Fries, 2005). When theta stimulation intensity was increased, this caused an increase in the phase locking of gamma cycles to their respective phases of the theta cycle, and therefore an increase in the precision and predictability of activity. This increase in the rigidity of the timing of gamma cycles during levels of high theta activity would enable the CA1 to increase its precision during times of high cognitive load, ensuring accurate communication between different cell assemblies.

\section{CA1 gamma is generated by a PING mechanism}

Through the use of both pharmacology and single-cell recordings, we have shown that intrinsic CA1 gamma is generated by a PING mechanism. Although ChR2 expression was not entirely limited to pyramidal neurons, it is unlikely that the small amount of ChR2 expressed in interneurons was important for the generation of CA1 gamma. Furthermore, changes in extracellular potassium levels can induce CA1 gamma oscillations (LeBeau et al., 2002), and we cannot rule out the possibility that extracellular potassium concentration is also important for the gamma oscillations recorded here. The pre-existing models of CA1 gamma are generated by a mixture of PING (Traub et al., 2003; Pietersen et al., 2014) and ING mechanisms (Whittington et al., 1995; Craig and McBain, 2015). Whether CA1 gamma in vivo is generated by a PING or ING mechanism is still not clear, although many studies have reported a delay in firing in CA1 interneurons compared with pyramidal cells consistent with the monosynaptic delay that is characteristic of PING (Bragin et al., 1995; Csicsvari et al., 2003; Senior et al., 2008; Korotkova et al., 2010).

Optogenetic technology has also been used in other in vitro models of gamma oscillations in the CAl's afferent regions, $\mathrm{mEC}$ and CA3. Indeed, Pastoll et al. (2013) demonstrated that optogenetic theta stimulation generated gamma oscillations in the $\mathrm{mEC}$ with similar properties to those observed here. This would suggest that the gamma generators present in the mEC and CA1 are analogous. Interestingly, the optogenetic mEC gamma was also phase-locked to the trough of the theta oscillation, and it remains to be seen whether conduction delays would result in propagated $\mathrm{mEC}$ gamma occurring at a different theta phase in CA 1 , as is the case in vivo (Schomburg et al., 2014).

Similarly, optogenetic stimulation of CA3 generated gamma oscillations of a similar frequency to those seen here (Akam et al., 2012), suggesting that another analogous generator is also pres- ent in CA3. This is also the case for pharmacological models, with in vitro CA3 (Fisahn et al., 1998) and CA1 gamma (Traub et al., 2003) having a similar frequency and slower than that of optogenetic gamma $(\sim 40 \mathrm{~Hz})$. These in vitro similarities make it likely that the different hippocampal gamma generators all share the same basic architecture and each have slight differences, such as the presence of recurrent excitation in $\mathrm{CA} 3$, that result in the differences in frequency of the different hippocampal gamma oscillations seen in vivo.

In summary, excitation of CA1 pyramidal neurons is sufficient to generate intrinsic CA1 gamma oscillations in vitro with similar properties to in vivo CA1 gamma oscillations. This model of CA1 gamma oscillations should therefore be useful to further our understanding of the complex interactions of distinct gamma oscillations present in CA1.

\section{References}

Ahmed OJ, Mehta MR (2012) Running speed alters the frequency of hippocampal gamma oscillations. J Neurosci 32:7373-7383. CrossRef Medline

Akam T, Oren I, Mantoan L, Ferenczi E, Kullmann DM (2012) Oscillatory dynamics in the hippocampus support dentate gyrus-CA3 coupling. Nat Neurosci 15:763-768. CrossRef Medline

Bartos M, Vida I, Jonas P (2007) Synaptic mechanisms of synchronized gamma oscillations in inhibitory interneuron networks. Nat Rev Neurosci 8:45-56. CrossRef Medline

Belluscio MA, Mizuseki K, Schmidt R, Kempter R, Buzsáki G (2012) Crossfrequency phase-phase coupling between $\theta$ and $\gamma$ oscillations in the hippocampus. J Neurosci 32:423-435.

Bragin A, Jandó G, Nádasdy Z, Hetke J, Wise K, Buzsáki G (1995) Gamma $(40-100 \mathrm{~Hz})$ oscillation in the hippocampus of the behaving rat. J Neurosci 15:47-60. Medline

Butler JL, Paulsen O (2015) Hippocampal network oscillations-recent insights from in vitro experiments. Curr Opin Neurobiol 31:40-44. CrossRef Medline

Buzsáki G, Wang XJ (2012) Mechanisms of gamma oscillations. Annu Rev Neurosci 35:203-225. CrossRef Medline

Buzsáki G, Leung LW, Vanderwolf CH (1983) Cellular bases of hippocampal EEG in the behaving rat. Brain Res 287:139-171. Medline

Chen Z, Resnik E, McFarland JM, Sakmann B, Mehta MR (2011) Speed controls the amplitude and timing of the hippocampal gamma rhythm. PLoS One 6:e21408. CrossRef Medline

Colgin LL, Denninger T, Fyhn M, Hafting T, Bonnevie T, Jensen O, Moser MB, Moser EI (2009) Frequency of gamma oscillations routes flow of information in the hippocampus. Nature 462:353-357. CrossRef Medline

Craig MT, McBain CJ (2015) Fast gamma oscillations are generated intrinsically in CA1 without the involvement of fast-spiking basket cells. J Neurosci 35:3616-3624. CrossRef Medline

Csicsvari J, Jamieson B, Wise KD, Buzsáki G (2003) Mechanisms of gamma oscillations in the hippocampus of the behaving rat. Neuron 37:311-322. CrossRef Medline

Fisahn A (1999) An investigation into cortical gamma frequency oscillations in vitro. $\mathrm{PhD}$ thesis, University of Oxford.

Fisahn A, Pike FG, Buhl EH, Paulsen O (1998) Cholinergic induction of network oscillations at $40 \mathrm{~Hz}$ in the hippocampus in vitro. Nature 394: 186-189. CrossRef Medline

Fries P (2005) A mechanism for cognitive dynamics: neuronal communication through neuronal coherence. Trends Cogn Sci 9:474-480. CrossRef Medline

Goutagny R, Krantic S (2013) Hippocampal oscillatory activity in Alzheimer's disease: toward the identification of early biomarkers? Aging Dis 4:134-140. Medline

Hájos N, Ellender TJ, Zemankovics R, Mann EO, Exley R, Cragg SJ, Freund TF, Paulsen O (2009) Maintaining network activity in submerged hippocampal slices: importance of oxygen supply. Eur J Neurosci 29: 319-327. CrossRef Medline

Korotkova T, Fuchs EC, Ponomarenko A, von Engelhardt J, Monyer H (2010) NMDA receptor ablation on parvalbumin-positive interneurons impairs hippocampal synchrony, spatial representations, and working memory. Neuron 68:557-569. CrossRef Medline 
Lasztóczi B, Klausberger T (2014) Layer-specific GABAergic control of distinct gamma oscillations in the CA1 hippocampus. Neuron 81: 1126-1139. CrossRef Medline

LeBeau FE, Towers SK, Traub RD, Whittington MA, Buhl EH (2002) Fast network oscillations induced by potassium transients in the rat hippocampus in vitro. J Physiol 542:167-179.

McFarland WL, Teitelbaum H, Hedges EK (1975) Relationship between hippocampal theta activity and running speed in the rat. J Comp Physiol Psychol 88:324-328. CrossRef Medline

Oren I, Hájos N, Paulsen O (2010) Identification of the current generator underlying cholinergically induced gamma frequency field potential oscillations in the hippocampal CA3 region. J Physiol 588:785-797. CrossRef Medline

Pastoll H, Solanka L, van Rossum MC, Nolan MF (2013) Feedback inhibition enables $\theta$-nested $\gamma$ oscillations and grid firing fields. Neuron 77: 141-154. CrossRef Medline

Paternain AV, Morales M, Lerma J (1995) Selective antagonism of AMPA receptors unmasks kainate receptor-mediated responses in hippocampal neurons. Neuron 14:185-189. CrossRef Medline

Peng H, Ruan Z, Long F, Simpson JH, Myers EW (2010) V3D enables realtime 3D visualization and quantitative analysis of large-scale biological image data sets. Nat Biotechnol 28:348-353. CrossRef Medline

Peng H, Bria A, Zhou Z, Iannello G, Long F (2014) Extensible visualization and analysis for multidimensional images using Vaa3D. Nat Protoc 9:193-208. CrossRef Medline

Pietersen AN, Ward PD, Hagger-Vaughan N, Wiggins J, Jefferys JG, Vreugdenhil M (2014) Transition between fast and slow gamma modes in rat hippocampus area $\mathrm{CA} 1$ in vitro is modulated by slow CA3 gamma oscillations. J Physiol 592:605-620. CrossRef Medline
Schomburg EW, Fernández-Ruiz A, Mizuseki K, Berényi A, Anastassiou CA, Koch C, Buzsáki G (2014) Theta phase segregation of input-specific gamma patterns in entorhinal-hippocampal networks. Neuron 84: 470-485. CrossRef Medline

Senior TJ, Huxter JR, Allen K, O’Neill J, Csicsvari J (2008) Gamma oscillatory firing reveals distinct populations of pyramidal cells in the CA1 region of the hippocampus. J Neurosci 28:2274-2286. CrossRef Medline

Sławińska U, Kasicki S (1998) The frequency of rat's hippocampal theta rhythm is related to the speed of locomotion. Brain Res 796:327-331. Medline

Stark E, Eichler R, Roux L, Fujisawa S, Rotstein HG, Buzsáki G (2013) Inhibition-induced theta resonance in cortical circuits. Neuron 80 : 1263-1276. CrossRef Medline

Traub RD, Cunningham MO, Gloveli T, LeBeau FE, Bibbig A, Buhl EH, Whittington MA (2003) GABA-enhanced collective behavior in neuronal axons underlies persistent gamma-frequency oscillations. Proc Natl Acad Sci U S A 100:11047-11052. CrossRef Medline

Uhlhaas PJ, Singer W (2010) Abnormal neural oscillations and synchrony in schizophrenia. Nat Rev Neurosci 11:100-113. CrossRef Medline

Whittington MA, Traub RD, Jefferys JG (1995) Synchronized oscillations in interneuron networks driven by metabotropic glutamate receptor activation. Nature 373:612-615. CrossRef Medline

Whittington MA, Traub RD, Kopell N, Ermentrout B, Buhl EH (2000) Inhibition-based rhythms: experimental and mathematical observations on network dynamics. Int J Psychophysiol 38:315-336. CrossRef Medline

Zemankovics R, Veres JM, Oren I, Hájos N (2013) Feedforward inhibition underlies the propagation of cholinergically induced gamma oscillations from hippocampal CA3 to CA1. J Neurosci 33:12337-12351. CrossRef Medline 